
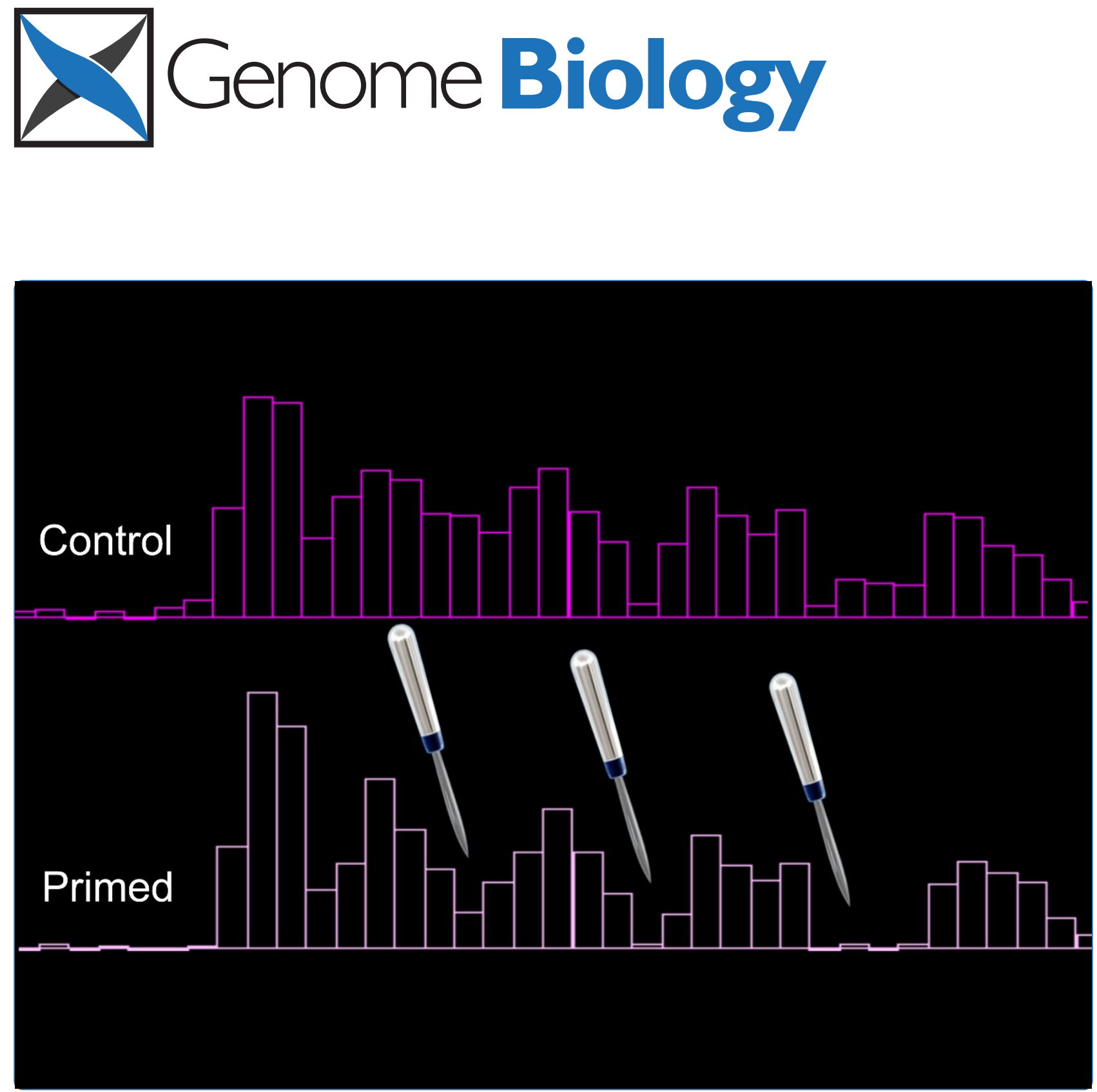

Hyperosmotic priming of Arabidopsis seedlings establishes a long-term somatic memory accompanied by specific changes of the epigenome 


\title{
Hyperosmotic priming of Arabidopsis seedlings establishes a long-term somatic memory accompanied by specific changes of the epigenome
}

\author{
Emanuela Sani ${ }^{1,3}$, Pawel Herzyk ${ }^{1}$, Giorgio Perrella ${ }^{1}$, Vincent Colot $^{2}$ and Anna Amtmann ${ }^{1 *}$
}

\begin{abstract}
Background: In arid and semi-arid environments, drought and soil salinity usually occur at the beginning and end of a plant's life cycle, offering a natural opportunity for the priming of young plants to enhance stress tolerance in mature plants. Chromatin marks, such as histone modifications, provide a potential molecular mechanism for priming plants to environmental stresses, but whether transient exposure of seedlings to hyperosmotic stress leads to chromatin changes that are maintained throughout vegetative growth remains unclear.

Results: We have established an effective protocol for hyperosmotic priming in the model plant Arabidopsis, which includes a transient mild salt treatment of seedlings followed by an extensive period of growth in control conditions. Primed plants are identical to non-primed plants in growth and development, yet they display reduced salt uptake and enhanced drought tolerance after a second stress exposure. ChIP-seq analysis of four histone modifications revealed that the priming treatment altered the epigenomic landscape; the changes were small but they were specific for the treated tissue, varied in number and direction depending on the modification, and preferentially targeted transcription factors. Notably, priming leads to shortening and fractionation of H3K27me3 islands. This effect fades over time, but is still apparent after a ten day growth period in control conditions. Several genes with priming-induced differences in H3K27me3 showed altered transcriptional responsiveness to the second stress treatment.
\end{abstract}

Conclusion: Experience of transient hyperosmotic stress by young plants is stored in a long-term somatic memory comprising differences of chromatin status, transcriptional responsiveness and whole plant physiology.

\section{Background}

Information storage (memory) is a prerequisite for the functioning of any biological or artificial system. Where and for how long information on experienced events should be stored depends on the purpose of the memory, such as altering immediate responses, learning through repetition, or archiving for future generations. Amount of information stored and duration of storage have to be carefully managed to avoid any negative impact on speed and fitness of the system.

\footnotetext{
* Correspondence: anna.amtmann@glasgow.ac.uk

'Institute of Molecular, Cell and Systems Biology (MCSB), College of Medical, Veterinary \& Life Sciences (MVLS), University of Glasgow, Glasgow G128QQ, UK

Full list of author information is available at the end of the article
}

Memory in higher plants is evident in altered responses to environmental stimuli after pre-exposure to the same or related stimuli, termed 'priming' or 'acclimation' depending on sequence and strength of the successive stimuli. Prominent examples include seed preconditioning, temperature acclimation, and systemic acquired resistance [1-3]. Over the last 10 years research into the molecular basis of plant memory has seen a boost of activity based on emerging knowledge on the role of chromatin modifications in determining gene activity $[4,5]$ and trait variation $[6,7]$.

Heritability of chromatin modifications through mitosis and meiosis provides a potential mechanism for long-term storage of information on environmental events both within the life span of an individual ('somatic memory')

\section{() Biomed Central}


and across generations ('trans-generational memory'). Several independent studies have reported chromatin changes (for example, loss of methylated DNA or of di-methylated lysine 9 in histone 3, H3K9me2) and re-activation of transposable elements in the offspring of environmentally challenged plants [8-12]. However, whether stress-induced chromatin changes are heritable through multiple generations and whether they underpin the acquisition of adaptive traits is still a matter of debate [13,14]. The evidence to date favors the view that stress-induced trans-generational changes of the chromatin might increase survival chances of the species, rather than each individual, by broadening the phenotypic plasticity and the genetic variation within the population [15-18].

Except for vernalization [19], the importance of chromatin modifications for long-term somatic memory of individuals is also still unclear, because in most studies carried out to date the effects of biotic or abiotic stimuli on chromatin were assessed either during or immediately after the priming treatment. Such experiments have provided evidence that environmental stimuli, such as pathogen attack [20-22] or drought [23-25], alter chromatin features, both genome-wide and at specific loci, but the consequences of these changes cannot easily be separated from other changes occurring at the same time. Given their potentially stable transmission through mitosis, and hence through growth, priminginduced chromatin marks could outlive changes in transcripts, proteins, hormones, and metabolites that will underlie more or less rapid turnover, but experimental evidence for this paradigm is sparse. In one case it was reported that a decrease of tri-methylated lysine 27 in histone 3 (H3K27me3) in the cold-responsive genes COR15A and ATGOLS3, caused by a short cold treatment of Arabidopsis thaliana seedlings, was still measurable 3 days after transferring the seedlings back into warm conditions although transcript levels had returned to control levels within $24 \mathrm{~h}$ [26]. These results have provided a first indication that H3K27me 3 could be a vehicle to translate transient transcriptional changes into a long-term memory but whether the identified marks are relevant for cold-acclimation remains to be investigated.

Another problem of comparative analysis of epigenetic profiles in environmentally challenged plants arises from confounding effects of plant growth and development. For example, prolonged application of drought and salt stress alter growth and development of the plants as well as inducing senescence and cell death in older leaf tissues. The phenotypic differences between treated and untreated plants complicate the interpretation of changes in histone modification profiles obtained from entire shoots because they will not only reflect stimulus-induced changes but also changes in the relative contributions of cell-line specific profiles to the overall organ profile [27].

In the study presented here we set out to develop an experimental protocol that allowed further investigation of the roles of histone modifications in long-term somatic memory. The prevailing concern was to apply a short and mild pre-treatment that had no apparent impact on plant growth and development, and to include a period of vegetative growth between the priming treatment and the second stress treatment that was long enough to ensure dilution and turnover of proteins and metabolites that were induced by the priming treatment. Within these constraints we had to prove that the chosen priming treatment was phenotypically effective and to test whether it caused durable changes in histone modifications. Drought and salinity were chosen as environmental stimuli because they represent a good example of a natural priming situation. Thus, in arid and semi-arid environments the vegetative growth period of plants is aligned to the wet season, and therefore exposure of plants to drought (and accompanying salinity) usually occurs early and late in plant life. However, whether exposure of young plants to drought/salinity enhances tolerance in adult plants has not been systematically tested in the laboratory. Based on the development of a controlled priming protocol we show here that a short treatment of young A. thaliana plants with $50 \mathrm{mM} \mathrm{NaCl}$ alters the response of adult plants to salt and drought even though both treatments are separated by an extensive period of growth in control conditions.

Four histone modifications were selected as molecular candidates for harboring a long-term somatic memory of salt stress. Di- or tri-methylated lysine 4 in histone 3 (H3K4me2 and H3K4me3) is enriched in transcriptionally active genes, and these two marks are particularly prominent among dehydration-responsive genes [23-25]. Furthermore, one of the enzymes catalyzing di- or trimethylation of H3K4 (ATX1) has been shown to be required for transcriptional and physiological responses A. thaliana to dehydration [28]. H3K27me3 and H3K9me2 are mutually exclusive chromatin modifications in A. thaliana [29], established and maintained by Polycomb complexes and SUVH histone methyltransferases, respectively [30]. Both marks have been associated with epigenetic inheritance of gene repression, but potential roles in long-term somatic memory of abiotic stress in plants have not yet been explored. Using ChIP-Seq and ChIP-qPCR we monitored genomewide profiles of all four modifications at high-resolution in primed and non-primed plants and identified specific alterations in the $\mathrm{H} 3 \mathrm{~K} 27 \mathrm{me} 3$ profile that were maintained over a 10-day growth period in control conditions. Our study therefore provides evidence for a 
long-term somatic memory in plants at the physiological and at the molecular level.

\section{Results and discussion}

\section{Hypersomotic priming at seedling stage alters stress} responses of adult plants

Based on a series of preliminary studies altering timing, strength, and duration of pre-treatments we developed an effective protocol for hyperosmotic priming of Arabidopsis thaliana plants including the following steps (Figure 1): Four-leaf stage seedlings grown on vertical agar plates received a 'priming treatment' consisting in the direct application of nutrient solution supplemented with $50 \mathrm{mM} \mathrm{NaCl}$ (priming) or not (control) to the roots. Twenty-four hours later all seedlings were transferred to soil or to hydroponics, and grown in control conditions for 10 days. During this period of time the plants increased their fresh weight by eight-fold in hydroponics and 15-fold in soil. A 'stress treatment' was then applied to both primed and non-primed plants either by withholding water from soil-grown plants (drought stress) or by adding $80 \mathrm{mM} \mathrm{NaCl}$ to hydroponically grown plants (salt stress). Primed and nonprimed plants were identical in size and appearance over the 10-day growth period (Figure 2A) and they did not differ in their sensitivity towards long-term (10 days) salt stress in hydroponics (Additional file 1, Figure S1). Nevertheless, over the first $24 \mathrm{~h}$ after salt application, primed plants accumulated significantly less $\mathrm{Na}$ in their shoots than non-primed plants (Figure 2B). Primed plants also showed a markedly higher tolerance to drought stress. Two weeks after onset of drought, nonprimed plants displayed strong symptoms of desiccation while primed plants were still green (Figure 2C). Biometric assessment of plants at an earlier stage of drought exposure revealed a dose-dependent effect of the priming treatment on plant growth under water-limited conditions (Figure 2D). One week after onset of drought, all plants were still green but weights and rosette diameters were larger in plants primed with 20 , 50 , or $100 \mathrm{mM} \mathrm{NaCl}$ than in non-primed plants. Best growth in water-limited conditions was achieved when $50 \mathrm{mM} \mathrm{NaCl}$ was used for priming, and this concentration was used for subsequent molecular analyses.

The results prove that plants have indeed a mechanism for long-term storage of information about transient exposure to a mild salt treatment at seedling stage, which allows them to respond better to a second stress exposure. The observed priming effects were in accordance with the notion that during the short priming treatment the seedlings experienced osmotic stress and increased $\mathrm{Na}$-influx but did not experience $\mathrm{Na}$-toxicity, which is the main stress factor during long-term exposure to high salt. Importantly, early exposure to a $24-\mathrm{h}$ mild salt treatment did not alter plant growth or development in unstressed conditions, and therefore any effects on subsequent stress responses must have been caused by molecular processes that did not impact on overall plant performance. Furthermore, any hormones, proteins, and metabolites generated in response to the priming treatment could be expected to underlie turnover and dilution during the growth period that

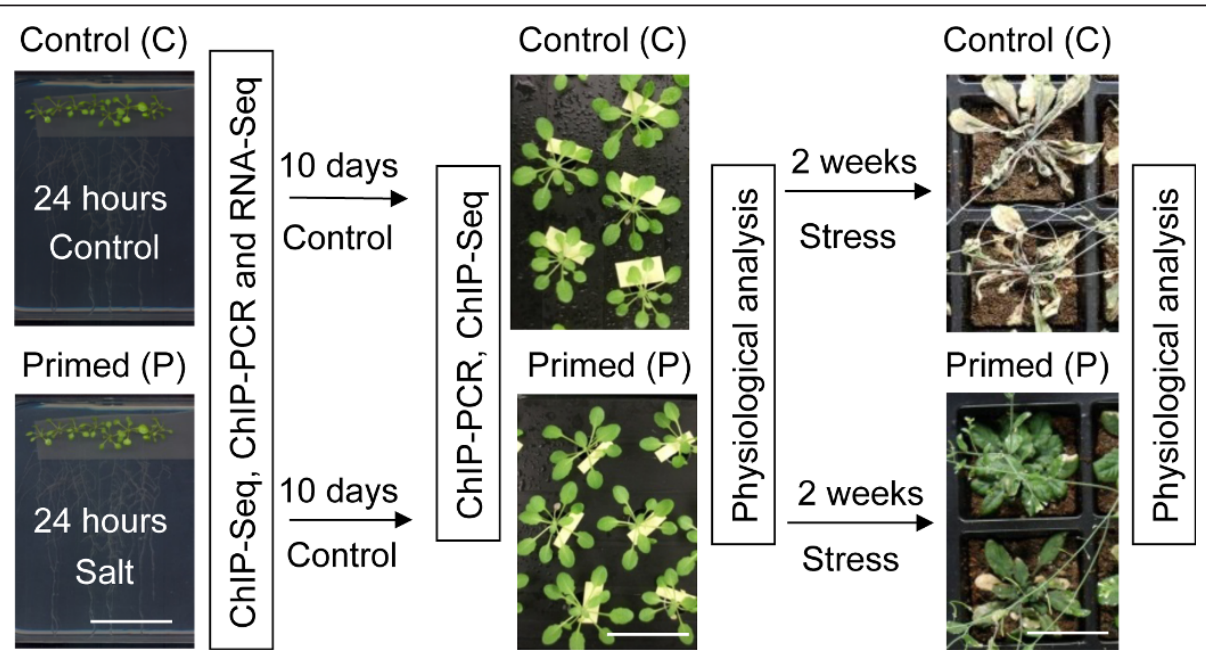

Figure 1 Experimental design to investigate somatic stress memory in A. thaliana. Arabidopsis thaliana plants were germinated on vertical agar plates. Growth medium supplemented with $\mathrm{NaCl}$ (or not, control) was applied directly to the roots (priming) of 3-week-old seedlings. After $24 \mathrm{~h}$ seedlings were transferred to hydroponics or to soil and grown for another 10 days without salt. A second treatment was then applied either by adding $\mathrm{NaCl}$ to the hydroponic solution (salt stress) or by withholding water from soil-grown plants. Epigenetic, transcriptional, and physiological differences between primed and non-primed plants were analyzed at the indicated times. Size bars in the photos: $4 \mathrm{~cm}$. 

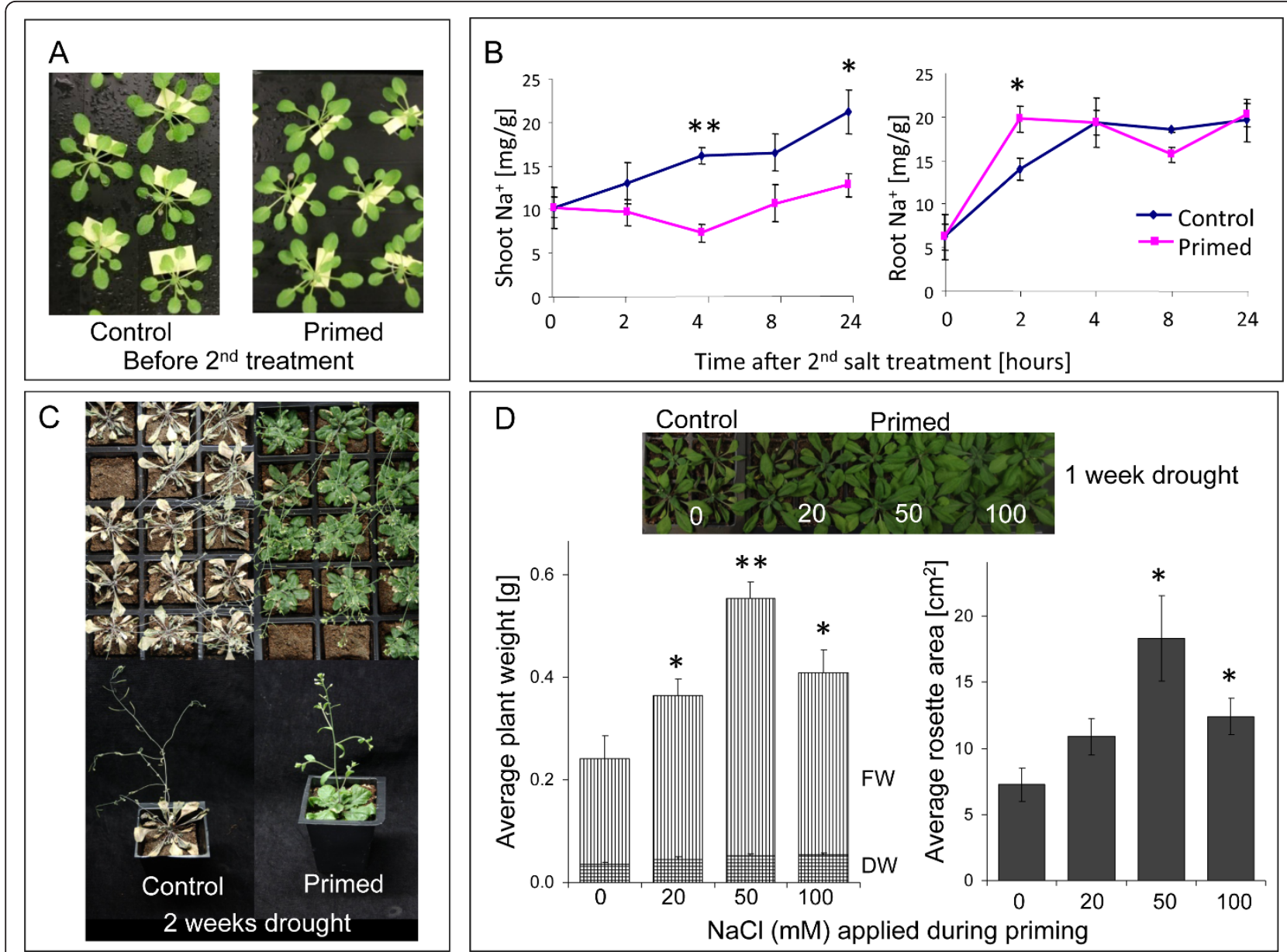

Figure 2 Salt priming at seedling stage alters responses of adult plants to salt and drought. (A) Appearance of primed and non-primed (control) plants after 10 days of growth in control conditions. Plants had been subjected to a 24-h treatment with 0 (control) or $50 \mathrm{mM} \mathrm{NaCl}$ (primed) at four-leaf seedling stage on agar plates, and subsequently transferred to hydroponics. (B) Shoot and root Na content in primed (pink) and non-primed (control, blue) plants after addition of $80 \mathrm{mM} \mathrm{NaCl}$ to the hydroponic solution of plants that had grown for 10 days in control conditions after priming. Means \pm SE of four individual plants are shown. Significant differences between primed and non-primed plants are indicated with * for $P<0.05$ and ${ }^{*}$ for $P<0.01$. (C) Appearance of primed and non-primed (control) plants 2 weeks after onset of drought stress. (D) Weight and seize of plants one week after onset of drought stress. Plants subjected to different concentrations of $\mathrm{NaCl}(0$ to $100 \mathrm{mM})$ during the priming treatment were analyzed. Dry weight (DW) is the horizontally dashed portion of fresh weight (FW) bars (vertically dashed). Each bar is the mean of 6-10 plants \pm SE. Significant differences between primed and non-primed plants are indicated with ${ }^{*}$ for $P<0.05$ and ${ }^{* *}$ for $P$ $<0.01$. Asterisks apply to both FW and DW.

separated the priming event from the second stress event. Chromatin marks were therefore the prime candidates for carrying the established memory.

\section{A mild hyperosmotic priming treatment alters histone modification profiles in seedlings}

To investigate whether the short, mild priming treatment had an effect on the epigenome, chromatin was isolated from the roots of primed and non-primed plants and immuno-precipitated with antibodies against histone modifications H3K4me2, H3K4me3, H3K9me2, and H3K27me3. Plants were harvested immediately after the 24-h priming (or control) treatment in three independent experiments each using approximately 300 plants per condition. Enrichment of histone modifications in previously identified regions of the genome was confirmed for each sample by qPCR ('quality control', Additional file 1, Figure S2) before each set of replicate samples was combined and sequenced by Illumina technology. Reads were counted over $200 \mathrm{bp}$ windows to obtain genomewide profiles ('landscapes') of the four modifications. Files containing read counts from all samples are provided as additional files 3 to 14 (see Methods) in a format that can be readily uploaded into the Integrated Genome Browser IGB [31]. At low resolution, the obtained histone modification landscapes reproduced the basic properties 
of previously published profiles, such as absence (H3K4me2, H3K4me3, H3K27me3) and presence (H3K9me2) of specific modifications around centromeres [32-34], and showed no obvious differences between primed and non-primed samples (Additional file 1, Figure S3). However, further analysis of the profiles at high resolution revealed several important differences between primed and non-primed samples, which will be described in the following.

Enriched histone modification domains ('islands') were determined with SICER software [35]. The highest number of islands (around 20,000) was identified for H3K4me2 and H3K4me3, followed by H3K27me3 (around 7,000) and H3K9me2 (around 2,000). Lists specifying the positions of identified islands in all samples are provided as Additional files 17 to 28 for upload into IGB (see Methods). The number of H3K4me2, $\mathrm{H} 3 \mathrm{~K} 4 \mathrm{me} 3$, and $\mathrm{H} 3 \mathrm{~K} 9 \mathrm{me} 2$ islands was similar in primed and non-primed plants, but the number of H3K27me3 islands increased from 6,288 in non-primed to 7,687 in primed plants (Figure 3A). The overall genome coverage with H3K4me2, H3K4me3, and H3K9me2 islands was again similar in primed and non-primed plants but genome coverage with H3K27me3 islands decreased from $19.3 \%$ in non-primed plants to $16.5 \%$ in primed plants despite the higher number of islands (Figure 3B).

\section{Priming induces small but specific changes in histone modification levels}

CHIPDIFF software [36] was used to identify genomic regions that differed in histone modification level between primed and non-primed samples. CHIPDIFF extracts only those sites that display differences between the two samples that are significantly larger than those in the neighboring regions thereby taking into account the overall noise in the profiles. Positions of all identified differences between primed and non-primed root samples are provided as additional files for upload into IGB (Additional files 31 to 36, see Methods). The number of identified differential sites depended on the lysine residue under consideration. H3K27me3 showed the largest number of differences, followed by H3K4me3 and H3K4me2, while H3K9me2 produced the least differences (Table 1). In most cases, the relative changes in the identified sites were small; only H3K27me3 displayed differences larger than two-fold. To investigate whether small differences in the H3K4me2 and H3K4me3 profiles could nevertheless be biologically meaningful we compared differences in the root samples to those in separately analyzed shoot samples of the same plants (primed root/non-primed roots and primed shoots/ non-primed shoots). Using a cutoff of 1.2-fold, we identified only 12 (H3K4me2) and 20 (H3K4me3) differences in shoots compared to 744 (H3K4me2) and
1,500 (H3K4me3) differences in roots (Table 1). Considering that roots but not shoots were in direct contact with the priming solution the finding provided strong support for a causal link between the priming treatment and the identified differences. At a higher cutoff (1.5fold) no differences were found in the shoot samples. The fact that high-resolution shoot profiles from primed and non-primed plants were virtually identical indicated that the pooling of $3 \times 300$ plants had eliminated treatment-independent variation within and between the replicate batches of plants.

The direction of priming-induced changes in methylation also depended on the specific lysine residue (Figure 3C). Only for H3K9me2 the number of differential sites that showed an increase in read count in primed plants was similar to the number of sites showing a decrease. For H3K4me2 and H3K4me3, the vast majority of identified differential sites showed a higher read count in the primed than in the non-primed sample, and many of the identified sites showed an increase in both di- and trimethylation of H3K4. By contrast, the vast majority of differential H3K27me3 sites showed a lower read count in the primed than in the non-primed sample. Opposite effects on H3K4 and H3K27 methylation consistently favor a more open chromatin structure in primed plants.

On the basis of the ChIP-Seq profiles we designed primer pairs within identified differential sites, and carried out qPCR using the ChIP samples from the individual replicate experiments. Dual normalization against input DNA and a constitutive reference region allowed direct quantitative comparison of the PCR amplifications. Figure 4 shows that qPCR faithfully reproduced the sitespecific small differences of H3K4me2, H3K4me3, and H3K27me3 that had been identified by CHIPDIFF in the genome-wide profiles. Priming-induced changes in H3K9me2 were not further analyzed in this study.

In summary, we found that the priming treatment did not lead to major remodeling of genome-wide histone modification profiles, but introduced small changes on top of the well-established landscapes of the four histone modifications tested. This could be expected since chromatin structure is essential for defining tissue identity and guiding developmental programs, and these fundamental functions should be robust against fluctuations in the environment. Indeed, the fact that we compared morphologically identical plants after a short, transient environmental stimulus sets our study apart from previous studies comparing plants that displayed stressinduced morphological differences after longer stress exposure. The small differences detected here could therefore be highly relevant for physiological differences between morphologically identical plants, and they would be suitable carriers of a long-term molecular memory that does not impact on plant development. 


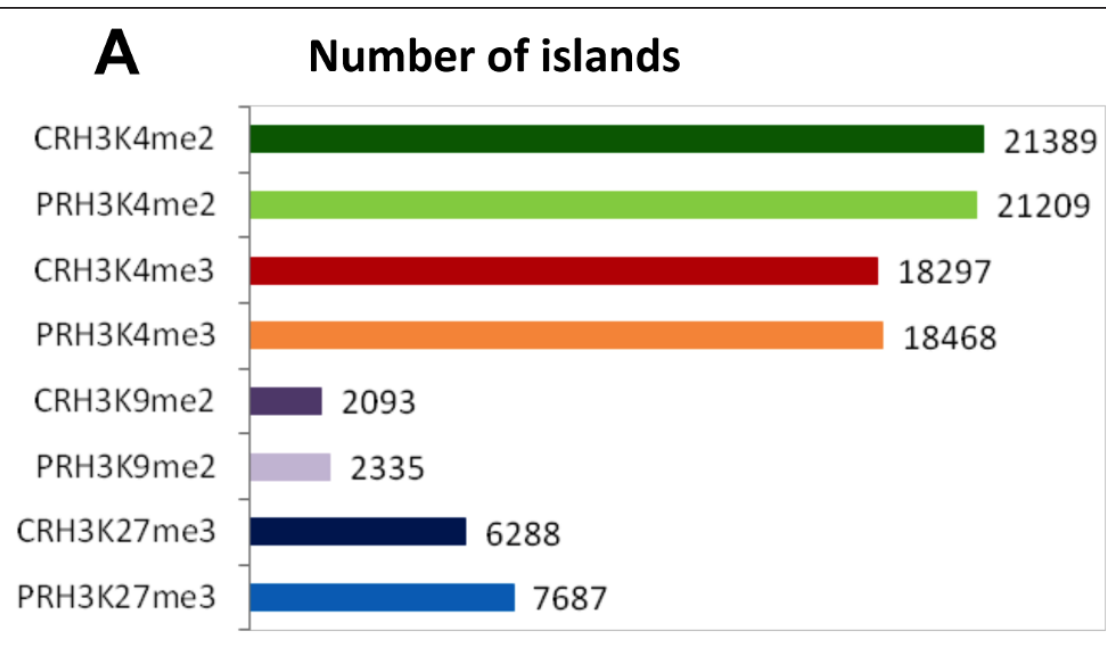

B \% genome coverage with islands

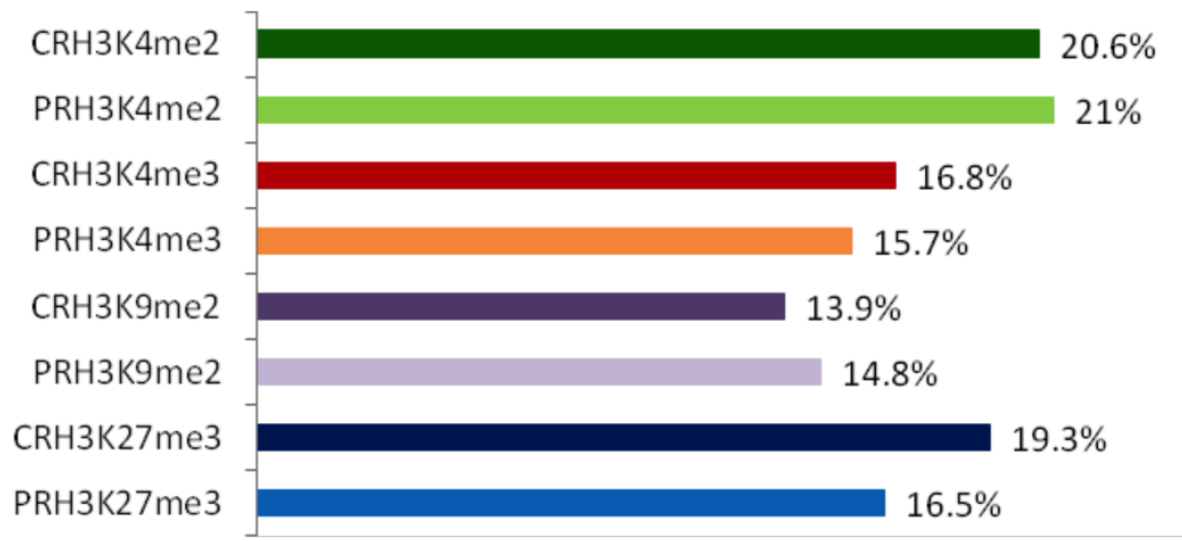

C Number and direction of differences

H3K4me2

H3K4me3

H3K9me2

H3K27me3 6520

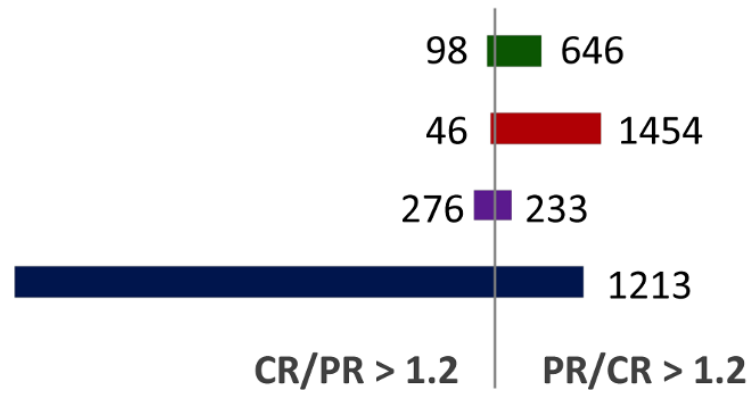

Figure 3 Effect of priming on genome-wide histone modification profiles. (A) Total number of continuous stretches of DNA ('islands') associated with specific histone modifications in roots of primed (PR; light colors) and non-primed (CR; dark colors) plants as determined by SICER [35]. (B) Total coverage of the genome with islands of specific histone modifications (in percent of whole genome sequence length). (C) Numbers of differences in genome-wide histone modification profiles identified by CHIPDIFF [36]. Numbers of differential sites that showed an increase of read count in the primed sample over the non-primed sample $(P R / C R>1.2)$ are plotted to the right those that showed a decrease $(C R / P R>1.2)$ are plotted to the left of the vertical bar. Data were obtained from pooled root material representing three independently treated plant batches of approximately 300 plants each. 
Table 1 Number of differences ${ }^{a}$ between primed and non-primed samples

\begin{tabular}{lllll}
\hline $\begin{array}{l}\text { Differences in roots } \\
(\boldsymbol{n})\end{array}$ & H3K4me2 & H3K4me3 & H3K9me2 & H3K27me3 \\
\hline$>$ 2-fold & 0 & 0 & 0 & 1678 \\
$>1.5$-fold & 71 & 105 & 10 & 3912 \\
$>1$-2-fold & 744 & 1500 & 509 & 7733 \\
\hline $\begin{array}{l}\text { Differences in shoots } \\
(n)\end{array}$ & H3K4me2 & H3K4me3 & H3K9me2 & H3K27me3 \\
\hline$>1.2$ fold & 12 & 20 & $n^{b}$ & nd $^{b}$ \\
\hline
\end{tabular}

adentified by CHIPDIFF.

${ }^{\mathrm{b}}$ Not determined.

\section{Priming 'etches' H3K27me3 islands}

Visual inspection of the histone modification profiles at high resolution revealed that differences in $\mathrm{H} 3 \mathrm{~K} 4 \mathrm{me} 2$ and H3K4me3 most commonly consisted in higher peaks of existing islands in the primed samples. By contrast, the majority of differences in $\mathrm{H} 3 \mathrm{~K} 27 \mathrm{me} 3$ occurred either at island edges or in 'valleys' within existing islands (see for example Figure 4). These sites already displayed low H3K27me3 occupancy in the nonprimed samples and occupancy was further reduced in the primed samples. Priming-induced shortening and fractionation ('etching') of existing H3K27me3 islands explained why a higher number of H3K27me3 islands were found to cover less of the genome (compare Figure $3 \mathrm{~A}, \mathrm{~B})$. Indeed island length distribution profiles of primed and non-primed samples (Figure 5) showed that the increase of $\mathrm{H} 3 \mathrm{~K} 27 \mathrm{me} 3$ island number in primed plants was only apparent in short islands (Figure 5A). Normalization to total island number revealed that the increase in the number of shorter H3K27me3 islands after priming was matched by a decrease in the number of long islands (Figure 5B). By contrast, island length

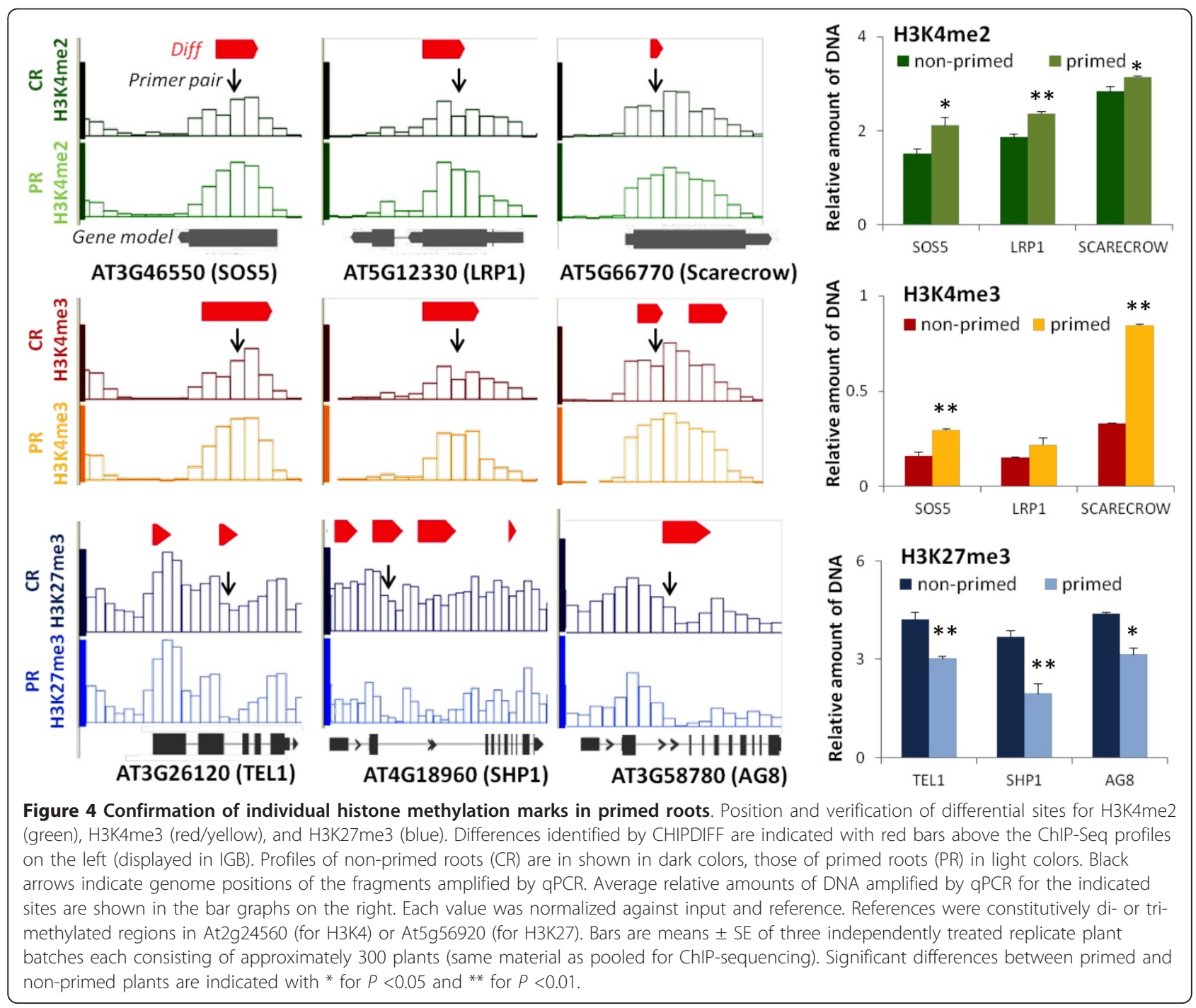




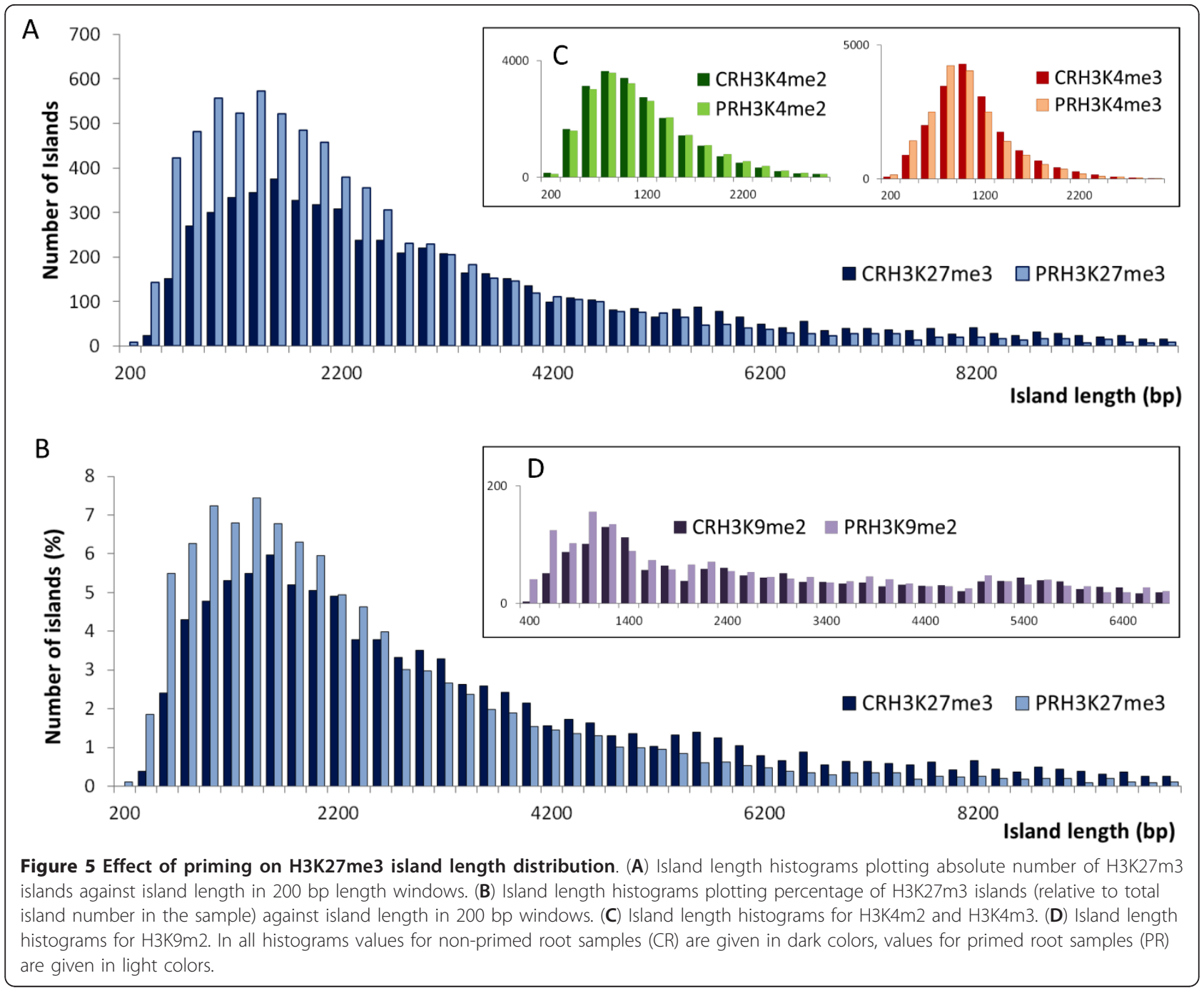

distributions of the other modifications were similar between primed and non-primed plants (Figure 5C, D).

The observation of H3K27me3 island etching is interesting because to date little is known about the spatial characteristics of H3K27me3 removal. Establishment of H3K27me3 is thought to occur through a 'nucleation' and 'spreading' process, which involves recruitment of PCR2 complex members first to specific sequences and then to the neighboring regions by an autocatalytic process $[37,38]$. Valleys within H3K27me 3 islands are then likely to reflect the merging of islands initiated at distinct nucleation sites. Here we found that a decrease of H3K27me3 in response to a mild salt treatment occurred primarily at island edges and in valleys within islands, thereby shortening existing islands and dividing longer islands into shorter ones. Thus removal of H3K27me3 appears to start from the youngest parts of the islands rather than the original nucleation regions thereby reversing the spreading of $\mathrm{H} 3 \mathrm{~K} 27 \mathrm{me} 3$. Considering that the changes were observed within $24 \mathrm{~h}$ we can assume that they are due to active demethylation rather than low maintenance of the mark during replication. The first and so far only enzyme shown to function as a H3K27me3 demethylase in plants has recently been identified as REF6 [39] but kinetic and spatial properties of REF6-mediated demethyation remain to be studied. A functional genetics approach should now be used to test whether REF6 is required for the priming-induced changes in H3K27me3 profiles, and for the physiological priming effects.

\section{Transcription factors are preferred targets of priming-} induced changes

To investigate which genes were targets of priminginduced changes we identified for all four modifications those islands and differential sites that mapped to genes 
resulting in gene lists for 'mapped islands' and 'mapped differences'. We then explored enrichment of functional annotations among genes carrying differential sites using the Database for Annotation, Visualization and Integrated Discovery (DAVID [40]). To compensate for any priming-independent functional bias among genes associated with certain histone modifications we scored enrichment of gene functions in 'mapped differences' not only over gene functions in the Arabidopsis genome (background 'Arabidopsis') but also over gene functions in the corresponding 'mapped islands' lists (backgrounds 'Islands Primed' and 'Islands Control'). The number of genes containing $\mathrm{H} 3 \mathrm{~K} 9 \mathrm{me} 2$ islands was too small to extract statistically significant results but a highly significant enrichment of genes encoding transcription factors was found among genes that experienced priminginduced changes in H3K4 and H3K27. The approximately two-fold enrichment of transcription factors was represented by several functional terms extracted from different databases, and was independent of the chosen background or the length of upstream and downstream sequences included in the mapping. An example of gene numbers, enrichment and statistical parameters for the term 'transcriptional regulation' is shown in Table 2. The full dataset is provided as Additional file 2. Our finding that priming-induced differences in $\mathrm{H} 3 \mathrm{~K} 27 \mathrm{me} 3$ were preferentially seen in transcription factors supports the notion of REF6 involvement because transcription factors were also found to be enriched among REF6binding genes [39].
Priming-induced changes in $\mathrm{H} 3 \mathrm{~K} 4 \mathrm{me} 3$ and $\mathrm{H} 3 \mathrm{~K} 27 \mathrm{me} 3$ do not merely reflect simultaneous transcriptional responses to the priming treatment

Plants respond to acute salt/hyperosmotic stress with changes in the transcription of many genes. We were interested whether the observed changes in H3K4me3 and H3K27me3 simply mirrored simultaneous transcriptional responses to the priming treatment. We therefore sequenced mRNA isolated from the same plant roots used for the ChIP-Seq experiments (harvested immediately after the $24-\mathrm{h}$ priming treatment). The mRNA sequence reads were mapped to the $A$. thaliana genome, and the mRNA levels obtained for each gene were plotted against cumulative read counts for H3K4me3 or H3K27me3 over the same genes, and ranked by mRNA level (Figure 6). Correlations were not apparent at single-gene level, but averaging over shifting windows of 200 genes reproduced the expected positive or negative correlation between gene expression and H3K4me3 or H3K27me3, respectively (Figure 6A, B). However, correlations were much weaker at high mRNA levels than at low mRNA levels, especially in the case of H3K27me3. Thus, mRNA levels of individual genes cannot be predicted from cumulative histone modification levels and vice versa. Furthermore, a lack of a dynamic relationship between gene activity and histone modification was apparent when the priming-induced changes in either parameter were compared for individual genes. As shown in Figure $6 \mathrm{C}$ the numbers of genes that showed changes in both mRNA level and histone modifications

Table 2 Enrichment ${ }^{a}$ of genes functionally classified as 'transcriptional regulation'b among genes that show priminginduced differences in $\mathrm{H} 3 \mathrm{~K} 4 \mathrm{me} 3$ or H3K27me3

\begin{tabular}{|c|c|c|c|c|c|c|}
\hline Modification & Sequence $^{c}$ & Background $^{d}$ & Count $^{\mathrm{e}}$ & $\%^{f}$ & Fold enrichment ${ }^{g}$ & $P$ value $^{\mathrm{h}}$ \\
\hline \multirow[t]{6}{*}{ H3K4me3 } & Gene $+1,000$ bp & Islands Primed & 129 & 8.86 & 1.98 & 4.97E-14 \\
\hline & & Islands Control & 127 & 8.72 & 2 & $4.32 \mathrm{E}-14$ \\
\hline & & Arabidopsis & 129 & 8.86 & 2.02 & $2.85 E-14$ \\
\hline & Gene + 100 bp & Islands Primed & 127 & 8.78 & 1.97 & $1.15 E-13$ \\
\hline & & Islands Control & 123 & 8.51 & 1.96 & $3.63 \mathrm{E}-13$ \\
\hline & & Arabidopsis & 129 & 8.92 & 2.04 & $1.73 \mathrm{E}-14$ \\
\hline \multirow[t]{6}{*}{ H3K27me3 } & Gene $+1,000 \mathrm{bp}$ & Islands Primed & 130 & 4.33 & 2.4 & $4.60 \mathrm{E}-21$ \\
\hline & & Islands Control & 139 & 4.63 & 1.4 & 5.05E-06 \\
\hline & & Arabidopsis & 238 & 7.94 & 1.83 & $3.88 \mathrm{E}-21$ \\
\hline & Gene + 100 bp & Islands Primed & 124 & 4.65 & 1.42 & $4.25 E-06$ \\
\hline & & Islands Control & 76 & 2.85 & 1.43 & 4.74E-04 \\
\hline & & Arabidopsis & 258 & 9.68 & 2.25 & $7.03 \mathrm{E}-37$ \\
\hline
\end{tabular}

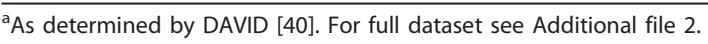

${ }^{\text {b}}$ Term from SP PIR KEYWORDS.

'Length of up- and downstream sequences included in the mapping of islands and differences to genes.

${ }^{d}$ Background list used for identification of enriched functional terms in submitted gene list.

${ }^{\mathrm{e}}$ Number of genes matching the annotation term in submitted gene list.

${ }^{f}$ Percentage of all genes in the submitted gene list.

${ }^{9}$ Relative enrichment compared to percentage in background.

${ }^{\mathrm{h}}$ Statistical significance of enrichment.
} 

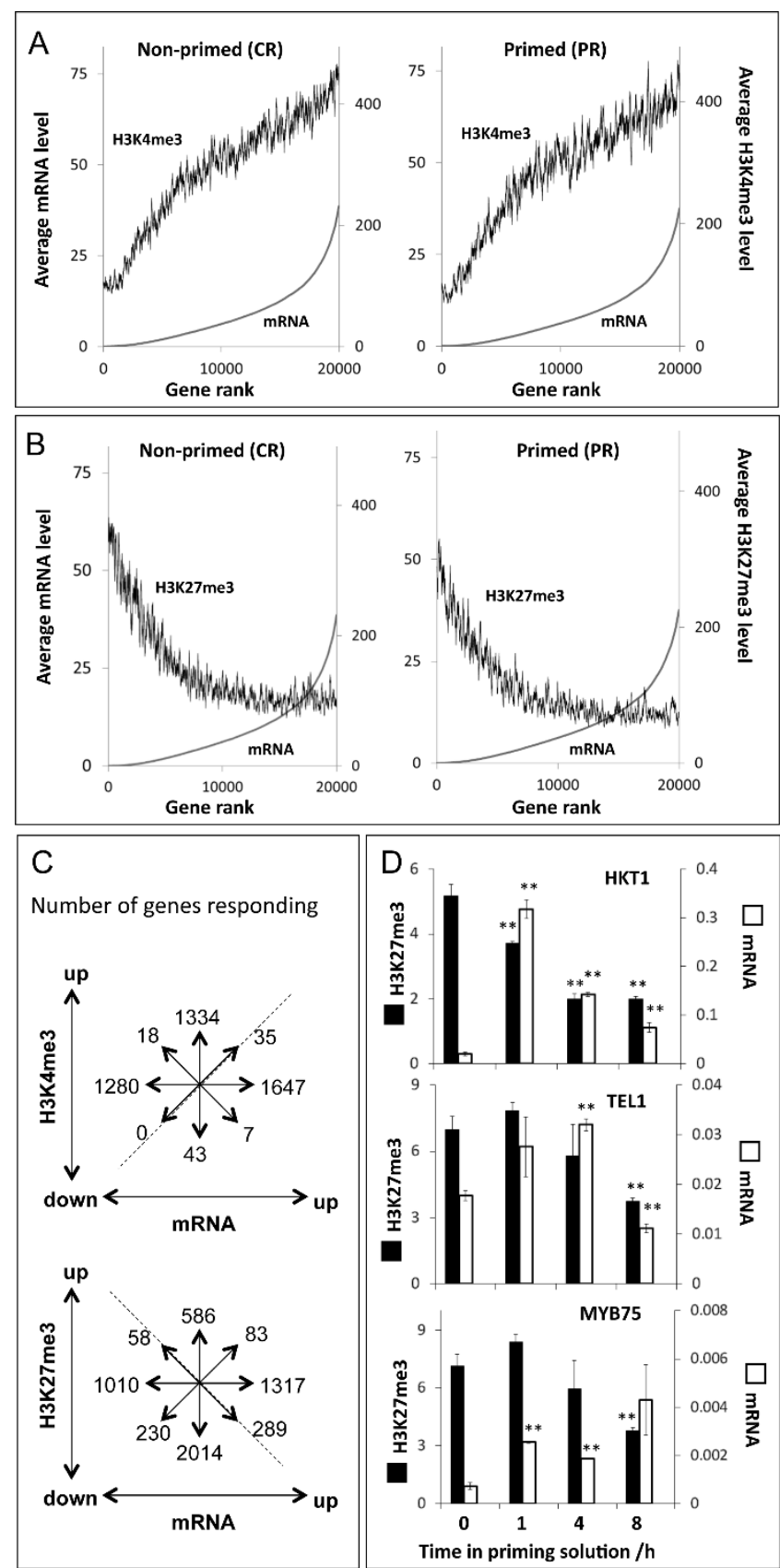

Figure 6 Relationship between histone methylation and mRNA levels during and after priming. (A, B) Genes on the $x$-axis were ranked according to mRNA levels determined by RNA-Seq. The mRNA profiles shown as smooth lines were generated from plotting for each gene on the $x$-axis the average mRNA values (right $y$-axis) over the neighboring genes with ranks of $+/-100$. Average values of histone modification levels (A: H3K4me3, B: H3K27me3) were plotted for the same genes (left y-axis). Relationships for non-primed root samples (CR) are shown in the graphs on the left, those for primed root samples (PR) are shown in the graphs on the right. (C) Numbers of genes that show an increase (up) or decrease (down) of mRNA level (x-axis) or histone modification level (y-axis) in response to the priming treatment (primed compared to nonprimed roots). Note that the majority of changes observed immediately after the priming treatment do not show the expected positive (H3K4me3) or negative (H3K27me3) correlation between mRNA and histone modification (dashed lines). (D) Short-term kinetics of changes of mRNA and H3K27me3 levels in three genes (HKT1, TEL1, and MYB75) during the priming treatment. Relative enrichment of H3K27me3 (black bars) and mRNA levels (open bars) of selected genes in roots of $A$. thaliana seedlings were determined by qPCR over a time course of the first 8 $\mathrm{h}$ (x-axis) of the priming treatment $(50 \mathrm{mM} \mathrm{NaCl})$. H3K27me3 enrichment (left $y$-axis) was normalized to ChIP input and to a reference region in At5g56920. mRNA levels (right y-axis) were normalized to reference gene Rpll. Bars show means \pm SE of four pairwise ratios of two technical replicates of qPCR carried out with pooled root material from approximately 50 plants per time point. Significant differences to time point 0 are indicated with * for $P<0.01$. 
after priming was low and the number of genes that showed the expected correlation was even lower. Our results are in contrast to those of a reported a strong positive correlation between changes of mRNA and H3K4me3 in drought-stressed plants [25]. However, in that study H3K4me3 and mRNA levels were measured several days after the onset of dehydration stress and thus reflect a new steady-state representing phenotypic differences in growth and development of the plants.

The observed lack of correlation between changes in mRNA and changes in chromatin modifications could be due to a difference in the kinetics of transcriptional regulation and chromatin biochemistry. For example, many transcriptional responses to salt are fast and transient [41], while chromatin changes may be slower and more persistent. We therefore analyzed by qPCR mRNA and $\mathrm{H} 3 \mathrm{~K} 27 \mathrm{me} 3$ levels of selected genes in roots of plants harvested during the first $8 \mathrm{~h}$ of the priming treatment. We found that changes in H3K27me3 were already detectable a few hours after salt addition. There was no consistent relationship between mRNA and H3K27me3 dynamics among the 10 genes analyzed (Additional file 1, Figure S4). However, for three genes (HKT1, TEL1, and MYB75) we found that rapid and transient induction at mRNA level was followed by a slower, long-lasting loss of H3K27me3 (Figure 6D).

To our knowledge, the kinetics of fast changes in histone modifications and mRNA immediately after a step change in the environment has not been explored before. Pulse-chase experiments in synchronized mammalian cells analyzed by mass spectrometry indicated that lysine tri-methylation is a relatively slow process requiring some $30 \mathrm{~h}$ to be re-established after DNA replication [42]. We found here that at least for some genes a decrease of H3K27me3 in response to a hyperosmotic stimulus already occurred within a few hours. This suggests that demethylation of H3K27me3 is a fast process that operates at a speed that is comparable to that of transcriptional regulation. Nevertheless the changes in specific H3K27me3 sites identified were in most cases not correlated with changes in mRNA, although in some genes they followed, and could have been triggered, by a fast transient change in transcription. Our findings underscore the fact that the exact relationship between histone modifications and transcription is still poorly understood. Both the reciprocal causality of chromatin modifications and transcriptional activity, and the temporal series of molecular events leading to changes in chromatin status are still under debate [43]. It will therefore be interesting to investigate in the future whether and how the changes in H3K27me3 observed here are mechanistically connected to other chromatin-based processes involved in dehydration-responsive gene transcription such as histone acetylation and/or nucleosome repositioning [44,45]. At this stage we conclude that a mild salt treatment causes rapid small changes in $\mathrm{H} 3 \mathrm{~K} 4 \mathrm{me} 3$ and $\mathrm{H} 3 \mathrm{~K} 27 \mathrm{me} 3$ that are superimposed onto the established steady-state correlation between transcript levels and histone modification status. The question then arises whether the priming-induced chromatin changes, once put into place, persist after removal of the original stimulus and whether they modulate gene transcription when the stimulus reoccurs.

\section{Priming-induced alterations of genome-wide H3K27me3 profiles are maintained during a 10-day growth period in control conditions}

To investigate whether priming-induced changes in the chromatin were maintained over the extensive growth period following the priming treatment, primed and non-primed plants were transferred to hydroponics and allowed to grow in control conditions for 10 days. At this point chromatin was isolated from roots of three independently grown plant batches and subjected to ChIP and quality control as before. This analysis was limited to H3K27me3, the modification that was most strongly affected by the priming. ChIP-qPCR analysis of selected genes showed that for five out of nine genes a decrease in H3K27me3 measured immediately after the 24-h priming treatment was still apparent 10 days later (Figure 7). For those genes that showed no longer a difference between primed and non-primed samples after 10 days, very little DNA was recovered from both 10 day samples (primed and non-primed), suggesting that a priming-independent loss of $\mathrm{H} 3 \mathrm{~K} 27 \mathrm{me} 3$ during plant maturation had cancelled the priming-induced mark. For HKT1 no DNA was recovered from the 10-day sample (see next section). Motivated by the qPCR results for individual genes, we pooled and sequenced the 10-day ChIP samples to identify long-term H3K27me3 changes at the genomic scale using the same methodology as before. All files obtained (aligned read counts, islands, and differences) are available as additional files for upload into IGB (Additional files 15, 16, 29, 30, and 37, see Methods). The total number of sequence reads from the 10-day samples was lower than from the 24-h samples and hence the landscapes had lost some of their depth. Nevertheless, the genome-wide profiles of the 10day samples reproduced the basic features discovered in the 24-h samples (Figure 8). For example, H3K27me3 islands occurred in larger number while covering less of the genome in primed plants than in non-primed plants (Figure 8, AB). Accordingly, the length distribution of H3K27me3 islands in primed samples was still skewed towards more, smaller islands in the primed samples (Figure $8 \mathrm{C}$ ), indicating that island fractionation was maintained during the growth period. Site-specific 

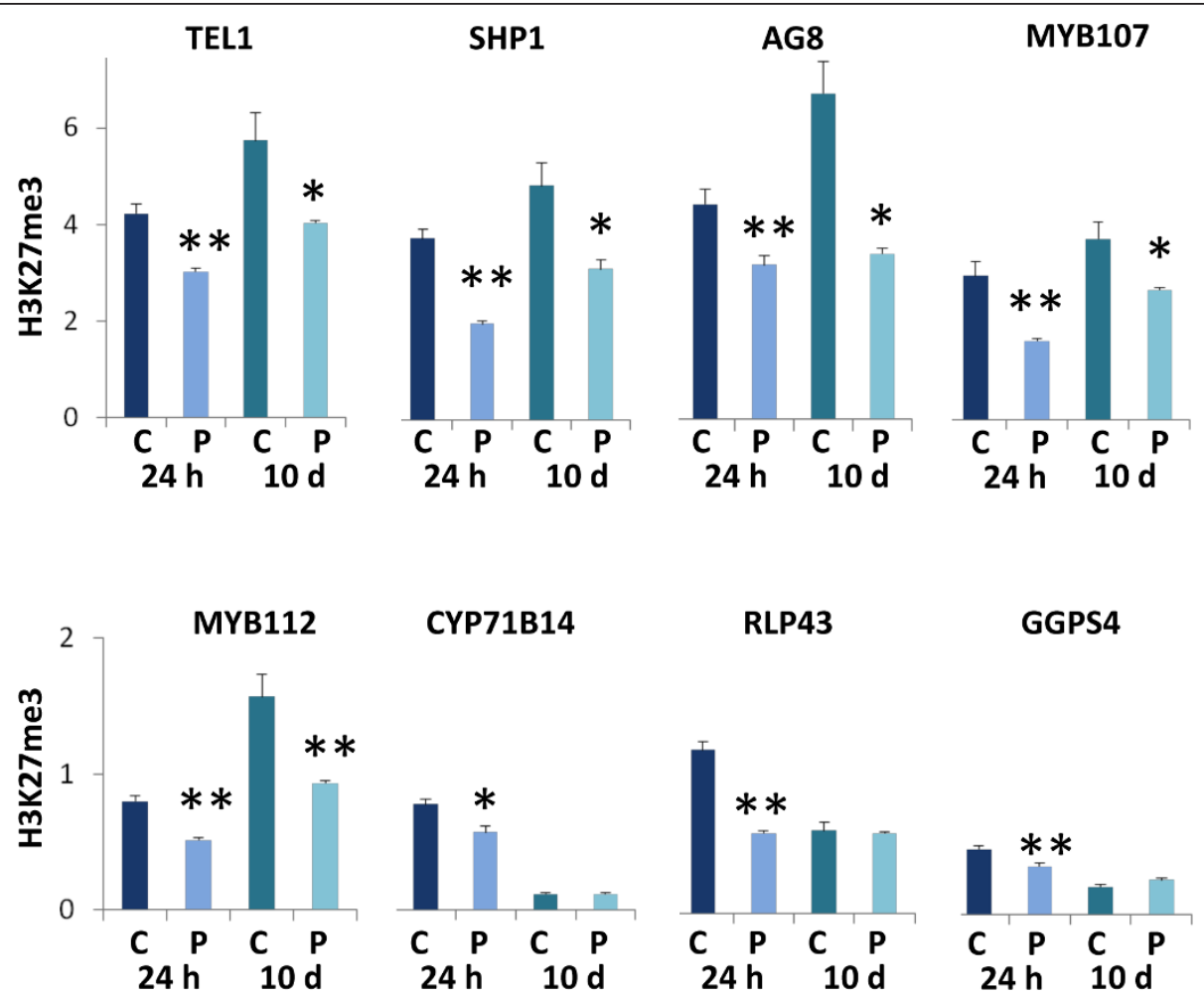

Figure 7 Maintenance and loss of H3K27me3 marks 10 days after priming. Average relative amounts of DNA amplified by qPCR from antiH3K27me3 ChIP samples obtained from roots of primed (P, dark color) and non-primed (C, light colors) plants immediately after the 24-h priming treatment ( $24 \mathrm{~h}$, blue) or 10 days later ( $10 \mathrm{~d}$, turquoise). Each value was normalized to ChIP input and to constitutive reference region in At5g56920. Bars are means \pm SE of three independently treated replicate plant batches each consisting of approximately 300 plants (same material as pooled for ChIP-sequencing). Significant differences between primed and non-primed plants are indicated with ${ }^{*}$ for $P<0.05$ and ${ }^{* *}$ for $P<0.01$.

differences between primed and non-primed samples, identified by CHIPDIFF, although lower in total number, still showed a bias towards a decrease of H3K27me3 in the primed plants (Figure 8D).

The genome-wide high resolution profiles revealed many cases of island etching at the same position in 24$\mathrm{h}$ and 10-day samples. Two examples are depicted in Figure 9. Comparison of positions of all differential sites between the 10-day and the 24-h samples resulted in a list of 102 genes with position-specific long-term changes in H3K27me3 (Table 3 and Additional file 1, Table S1). Interestingly, in many cases the length of the affected region was shorter after the 10-day growth period than immediately after the priming treatment. We conclude that the gaps in H3K27me3 islands that were generated by the priming treatment were transmitted through mitosis, but progressively 'filled' during growth in non-stressed conditions, probably due to PRC2mediated spreading of $\mathrm{H} 3 \mathrm{~K} 27 \mathrm{me} 3$ into the etched areas. Thus, priming-triggered demethylation of $\mathrm{H} 3 \mathrm{~K} 27$ at and within existing islands might require active maintenance in order to prevent fading of the molecular memory through H3K27me3 spreading. It would be interesting to investigate now whether repeated exposure to salt can prevent memory loss and eventually lead to the removal of entire H3K27me3 islands.

Priming alters the transcriptional response of the sodium transporter HKT1 to salt stress after a 10-day growth period in control conditions

The observation of a marked loss of H3K27me 3 in HKT1 (At4g10310) during the priming treatment (Figure 6D) attracted our attention. HKT1 is a root-specific $\mathrm{Na}$ transporter that removes $\mathrm{Na}$ from the transpiration stream [46]. Indeed, lower shoot $\mathrm{Na}$ accumulation in primed plants after application of the second salt treatment (Figure 2B) mimicked the phenotype of A. thaliana mutant lines over-expressing HKT1 specifically in xylem parenchyma cells [47]. We therefore measured mRNA levels of HKT1 after application of the second salt treatment 10 days after the priming treatment. In accordance with previous findings, HKT1 displayed root specific expression in all plants. Importantly, in three independently primed plant batches HKT1 mRNA was 


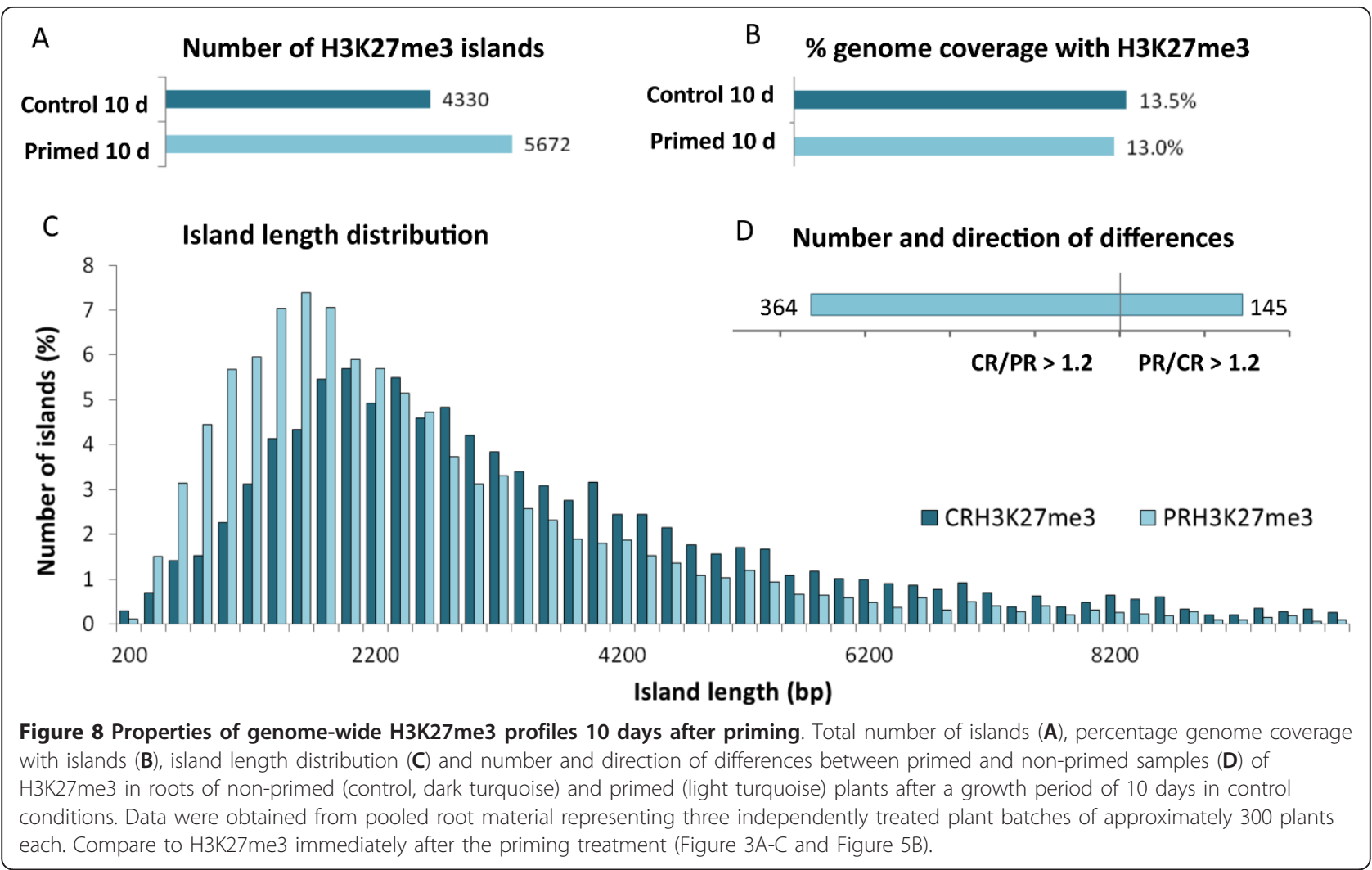

always most abundant in the salt-treated primed plants (Figure 9). This was either due to a higher constitutive level (Figure 9A, replicate 1) or to stronger induction by salt in primed plants (Figure 9A, replicates 2 and 3). The experiment not only showed that the priming treatment still affected transcriptional responses after the 10day growth period in control conditions, but also identified HKT1 as prime candidate for explaining at least one the physiological effects of the priming treatment. We were not able to recover enough DNA from antiH3K27me3 ChIP from the individual replicate 10-day samples to allow detection of H3K27me3 in the differential HKT1 site by qPCR. However, the high-resolution profile obtained by ChIP-sequencing, while confirming very low H3K27me3 occupancy in the mature plants, still showed lower read counts in the primed sample than in the non-primed sample after 10 days (Figure 9B). The findings suggest that a persistent loss of H3K27me3 at HKT1 occurs in a very small number of cells that occupy a strategically important position in the root while HKT1 is silenced through other mechanisms in the rest of the plant. Indeed, HKT1 fulfills different functions depending on developmental stage and cell type $[48,49]$, with shoot $\mathrm{Na}$ limitation being a consequence of HKT1 activity in root xylem parenchyma cells [47]. It is therefore conceivable that in adult plants H3K27me3 occupancy of HKT1 and changes thereof are also cell-type specific. Silencing of HKT1 in the shoots has been linked to distantly upstream tandem repeats and to siRNA-mediated DNA methylation within the HKT1 promoter [50,51]. Similar processes might silence HKT1 in most root cells of adult plants and preclude H3K27me3 occupancy. In this case very few cells would have contributed DNA to the anti-H3K27me3 ChIP sample thereby explaining low read count in the 10-day samples. Cell-type specific ChIP experiments are now needed to further consolidate the role of HKT1 in somatic salt stress memory.

\section{Several other genes also show altered transcriptional} responses to the second salt treatment

Although over-expression of HKT1 alone could explain the low-sodium phenotype of primed plants, the 4-h salt treatment also represented an opportunity to investigate early transcriptional responses to a hyperosmotic stimulus. We therefore used the root mRNA samples from this treatment and time point to test for priming-dependent transcriptional responses of other genes. Twenty genes covering a range of functions were selected from Table 3 for qPCR analysis. Most of these genes had very low expression levels in both primed and non-primed plants independent of whether they were treated with salt or not (Additional file 1, Table S2). However, we found that PIP2E (At2g39010), encoding a plasma 


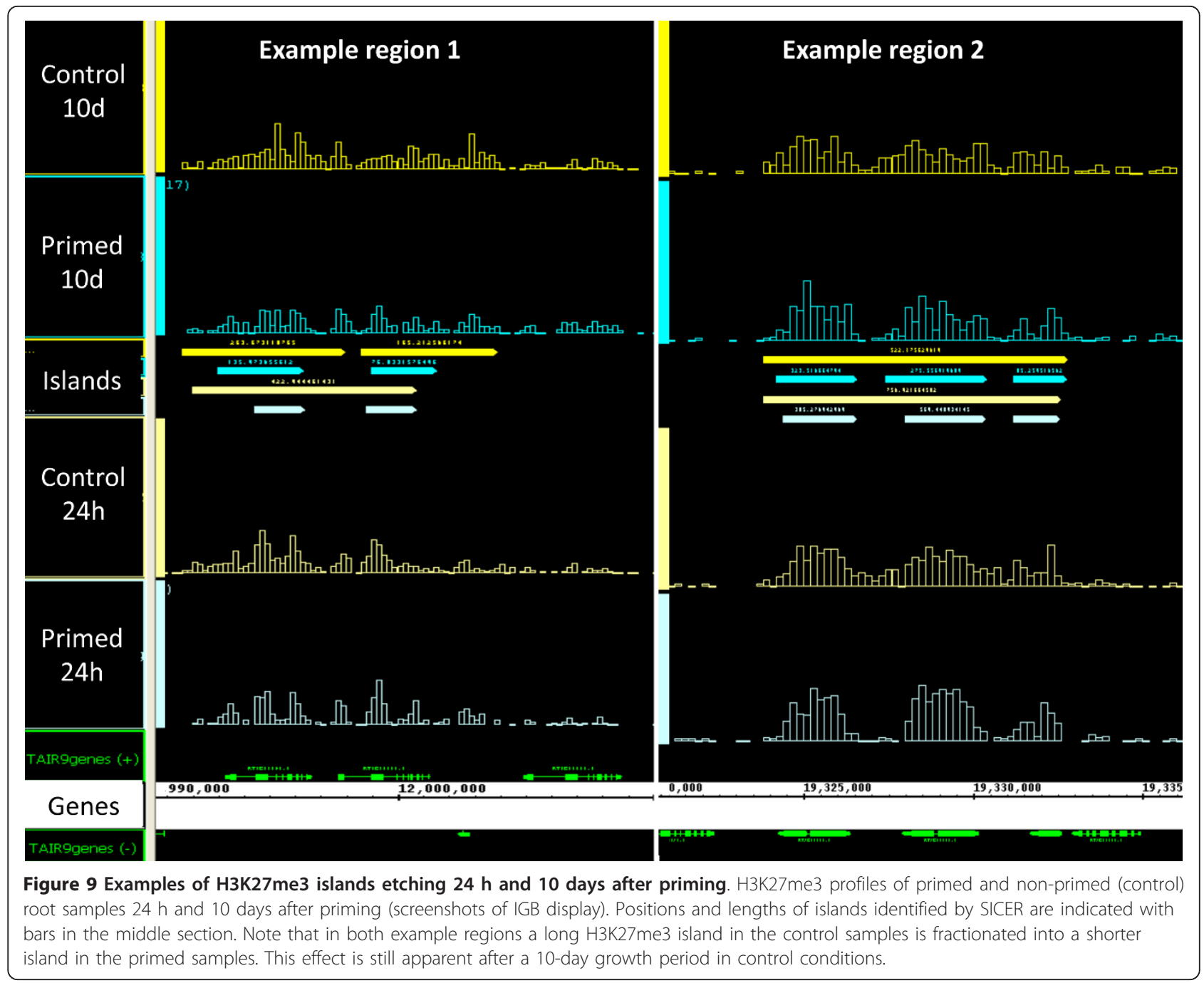

membrane aquaporin [52], was upregulated in the salttreated plants and that this response was stronger in the primed than in the non-primed plants. Two other genes, GH3.1 (At2g14960) and GH3.3 (At2g23170), encoding auxin and jasmonate amino-acid conjugating enzymes respectively [53], were also upregulated by the salt treatment, but showed a weaker response in primed than in non-primed plants. Opposite effects of priming on the transcriptional responsiveness of PIP2E and HKT1 (more responsive) and on GH3.1 and GH3.3 (less responsive) were in accordance with opposite effects of priming on H3K27me3 in these genes, with PIP2E and HKT1 experiencing a decrease of H3K27me3 and GH3.1 and GH3.3 experiencing an increase. Whether this correlation reflects a direct causal relationship between H3K27me3 levels and transcriptional regulation during the second stress response needs now to be further investigated. Our results support the view that the priming-induced chromatin changes do not affect constitutive transcriptional activity but modulate access of stress-inducible transcriptional regulators thereby limiting any priming effects to reoccurring stress situations. The exact contributions of the identified differentially regulated genes to the physiological priming effect remain to be identified but they are likely to be related to water and hormone homeostasis during osmotic stress. Interestingly, GH3.1 is exclusively expressed in the root epidermis and hence in direct contact with the root environment [54].

In summary, we have identified four genes that show persistent priming-induced changes of H3K4me3 and altered transcriptional responses in response to a second salt treatment. Clearly this is just the beginning of a wider search for genes that could link the epigenetic memory to the physiological priming effects. This search needs to cover a range of stimuli and time points during the second stress exposure. In particular, it will be necessary to design a soil-free 'drought' experiment that 
Table 3 Direction and length of priming-induced stable differences in H3K27me3

\begin{tabular}{|c|c|c|c|c|c|}
\hline Gene ID & Description (from TAIR) & $\begin{array}{l}P / C^{\mathrm{a}} \\
24 \mathrm{~h}^{\mathrm{b}}\end{array}$ & Length $^{\mathrm{c}} 24 \mathrm{~h} / \mathrm{bp}$ & Length $10 \mathrm{~d} / \mathrm{bp}$ & $\begin{array}{l}P / C^{a} \\
10 d^{d}\end{array}$ \\
\hline AT1G05291 & Unknown protein & Down & 200 & 200 & Down \\
\hline AT1G09380 & Integral membrane family/MtN21-related & Down & 200 & 200 & Down \\
\hline AT1G12190 & F-box family protein & Down & 800 & 400 & Down \\
\hline AT1G12260 & ANAC007; transcription factor & Down & 600 & 400 & Down \\
\hline AT1G18710 & AtMYB47 transcription factor & Down & 600 & 200 & Down \\
\hline AT1G19800 & TGD1; lipid transporter & Down & 200 & 200 & Down \\
\hline AT1G30795 & Hydroxyproline-rich glycoprotein family protein & Down & 200 & 200 & Down \\
\hline AT1G31875 & Unknown protein & Down & 600 & 200 & Down \\
\hline AT1G47370 & Toll-Interleukin-Resistance (TIR) domain & Down & 600 & 200 & Down \\
\hline AT1G47786 & Acyl-protein thioesterase-related & Down & 400 & 200 & Down \\
\hline AT1G51460 & $\mathrm{ABC}$ transporter family protein & Down & 600 & 200 & Down \\
\hline AT1G51490 & BGLU36 (beta glucosidase 36); hydrolase & Down & 200 & 400 & Down \\
\hline AT1G52070 & Jacalin lectin family protein & Down & 200 & 200 & Down \\
\hline AT1G52140 & Unknown protein & Down & 1,800 & 200 & Down \\
\hline AT1G52410 & TSA1; calcium ion binding/protein binding & Down & 800 & 200 & Down \\
\hline AT1G56650 & PAP1; transcription factor & Down & 600 & 200 & Down \\
\hline AT1G57830 & Toll-Interleukin-Resistance (TIR) domain & Down & 400 & 400 & Down \\
\hline AT1G59722 & Unknown protein & Down & 1,000 & 200 & Down \\
\hline AT1G61630 & ENT7 equilibrative nucleoside transporter & Down & 800 & 600 & Down \\
\hline AT1G61750 & Molecular_function unknown & Down & 200 & 200 & Down \\
\hline AT1G62030 & DC1 domain-containing protein & Up & 200 & 400 & Up \\
\hline AT1G65342 & Unknown protein & Down & 200 & 200 & Down \\
\hline AT1G67710 & ARR11; transcription factor/ two-component & Down & 400 & 200 & Down \\
\hline AT1G69090 & F-box family protein & Down & 800 & 200 & Down \\
\hline AT1G69120 & AP1 (Apetala 1); DNA binding & Down & 800 & 200 & Down \\
\hline AT1G75920 & Family II extracellular lipase 5 (EXL5) & Down & 400 & 200 & Down \\
\hline AT2G14710 & F-box family protein & Down & 200 & 200 & Down \\
\hline AT2G14960 & $\mathrm{GH} 3.1$ & Up & 400 & 200 & Up \\
\hline AT2G16220 & F-box family protein & Down & 600 & 200 & Down \\
\hline AT2G21890 & CAD3 cinnamyl alcohol dehydrogenase & Down & 400 & 400 & Down \\
\hline AT2G22180 & Hydroxyproline-rich glycoprotein family protein & Down & 400 & 200 & Down \\
\hline AT2G23170 & GH3.3; indole-3-acetic acid amido synthetase & Up & 400 & 200 & Up \\
\hline AT2G23171 & Unknown protein & Down & 200 & 200 & Down \\
\hline AT2G24205 & ECA1 gametogenesis related family protein & Down & 200 & 200 & Down \\
\hline AT2G25697 & Unknown protein & Down & 600 & 200 & Down \\
\hline AT2G25700 & ASK3 (SKP1-like 3); ubiquitin-protein ligase & Down & 600 & 200 & Down \\
\hline AT2G26580 & YAB5 (YABBY5); transcription factor & Down & 600 & 1,000 & Down \\
\hline AT2G30300 & Nodulin-related & Down & 200 & 200 & Down \\
\hline AT2G30760 & Unknown protein & Down & 200 & 200 & Down \\
\hline AT2G31083 & CLE5 (Clavata3/ESR-related 5) & Down & 1,200 & 200 & Down \\
\hline AT2G32870 & Meprin and TRAF homology domain protein & Down & 200 & 200 & Down \\
\hline AT2G34790 & MEE23; FAD binding & Down & 800 & 200 & Down \\
\hline AT2G39010 & PIP2E; water channel & Down & 600 & 400 & Down \\
\hline AT3G03200 & Anac045; transcription factor & Down & 400 & 200 & Down \\
\hline AT3G09390 & MT2A; copper ion binding & Down & 400 & 400 & Down \\
\hline AT3G16360 & AHP4; histidine phosphotransfer kinase & Down & 200 & 200 & Down \\
\hline AT3G18010 & WOX1 (Wuschel related); transcription factor & Down & 600 & 200 & Down \\
\hline AT3G18550 & BRC1 (Branched 1); transcription factor & Down & 1,800 & 200 & Down \\
\hline AT3G20160 & Geranylgeranyl pyrophosphate synthase, putative & Up & 600 & 200 & Up \\
\hline AT3G21840 & ASK7 (SKP1-LIKE 7); ubiquitin-protein ligase & Down & 400 & 400 & Down \\
\hline AT3G21850 & ASK9 (SKP1-LIKE 9); ubiquitin-protein ligase & Down & 400 & 200 & Down \\
\hline AT3G22080 & Meprin and TRAF homology domain protein & Down & 200 & 200 & Down \\
\hline
\end{tabular}


Table 3 Direction and length of priming-induced stable differences in H3K27me3 (Continued)

\begin{tabular}{|c|c|c|c|c|c|}
\hline AT3G25710 & BHLH32; transcription factor & Down & 600 & 200 & Down \\
\hline AT3G29260 & Short-chain dehydrogenase/reductase family & Down & 800 & 200 & Down \\
\hline AT3G29970 & Germination protein-related & Down & 800 & 400 & Down \\
\hline AT3G44780 & Unknown protein & Down & 1,000 & 200 & Down \\
\hline AT3G45560 & Zinc finger (C3HC4-type RING finger) family & Down & 600 & 200 & Down \\
\hline AT3G50480 & HR4 (homolog of RPW8 4) & Down & 600 & 200 & Down \\
\hline AT3G51200 & Auxin-responsive family protein & Down & 400 & 200 & Down \\
\hline AT3G55700 & UDP-glucoronosyl/UDP-glucosyl transferase & Down & 200 & 400 & Down \\
\hline AT4G00300 & Fringe-related protein & Up & 400 & 200 & Up \\
\hline AT4G01420 & CBL5 (Calcineurin B-like protein 5) & Down & 400 & 200 & Down \\
\hline AT4G01520 & Anac067; transcription factor & Down & 200 & 200 & Down \\
\hline AT4G04840 & Methionine sulfoxide reductase domain protein & Down & 1,000 & 200 & Down \\
\hline AT4G04890 & PDF2; transcription factor & Down & 800 & 200 & Down \\
\hline AT4G10220 & Unknown protein & Down & 1,000 & 200 & Down \\
\hline AT4G10350 & ANAC070; transcription factor & Down & 400 & 200 & Down \\
\hline AT4G11170 & Disease resistance protein (TIR-NBS-LRR class) & Down & 1,200 & 200 & Down \\
\hline AT4G12510 & Lipid transfer protein (LTP) family protein & Down & 600 & 400 & Down \\
\hline AT4G12520 & Lipid transfer protein (LTP) family protein & Down & 400 & 200 & Down \\
\hline AT4G13420 & HAK5 (high affinity K+ transporter 5) & Up & 400 & 200 & Up \\
\hline AT4G19730 & Glycosyl hydrolase family 18 protein & Down & 600 & 400 & Down \\
\hline AT4G19740 & Catalytic/ cation binding/chitinase/ hydrolase & Down & 1,600 & 200 & Down \\
\hline AT4G22030 & F-box family protein & Down & 200 & 200 & Down \\
\hline AT4G22070 & WRKY31; transcription factor & Down & 200 & 200 & Down \\
\hline AT4G28840 & Unknown protein & Down & 800 & 200 & Down \\
\hline AT4G29033 & Encodes a defensin-like (DEFL) family protein. & Down & 800 & 200 & Down \\
\hline AT4G30590 & Plastocyanin-like domain-containing protein & Down & 200 & 200 & Down \\
\hline AT5G04386 & Unknown protein & Down & 200 & 200 & Down \\
\hline AT5G11520 & ASP3 (aspartate aminotransferase 3) & Down & 400 & 400 & Down \\
\hline AT5G15160 & bHLH family protein & Down & 600 & 200 & Down \\
\hline AT5G15210 & ATHB30; transcription factor & Up & 200 & 400 & Up \\
\hline AT5G15800 & SEP1 (Sepallata 1); transcription factor & Down & 600 & 200 & Down \\
\hline AT5G17100 & Molecular_function unknown & Down & 400 & 400 & Down \\
\hline AT5G17810 & WOX12 (Wuschel related); transcription factor & Down & 1,600 & 600 & Down \\
\hline AT5G17960 & DC1 domain-containing protein & Up & 1,000 & 200 & Down \\
\hline AT5G24070 & Peroxidase family protein & Down & 200 & 200 & Down \\
\hline AT5G24820 & Aspartyl protease family protein & Up & 200 & 400 & Up \\
\hline AT5G24910 & CYP714A1; electron carrier/ heme binding & Down & 200 & 400 & Down \\
\hline AT5G25390 & SHN2 (shine2); transcription factor & Down & 1,000 & 200 & Down \\
\hline AT5G25990 & Unknown protein & Down & 200 & 200 & Down \\
\hline AT5G35770 & SAP (sterile apetala); transcription factor & Down & 1,000 & 400 & Down \\
\hline AT5G39560 & Molecular_function unknown & Up & 600 & 200 & Up \\
\hline AT5G40040 & $60 S$ acidic ribosomal protein P2 (RPP2E) & Up & 400 & 200 & Up \\
\hline AT5G40790 & Unknown protein & Down & 800 & 200 & Down \\
\hline AT5G42590 & CYP71A16; electron carrier/ heme binding & Down & 600 & 200 & Down \\
\hline AT5G43120 & Tetratricopeptide repeat (TPR)-containing protein & Up & 400 & 400 & Up \\
\hline AT5G45200 & Disease resistance protein (TIR-NBS-LRR class) & Down & 1,000 & 200 & Down \\
\hline AT5G46350 & WRKY8; transcription factor & Down & 400 & 400 & Down \\
\hline AT5G57785 & Unknown protein & Down & 800 & 400 & Down \\
\hline AT5G60010 & FAD binding/calcium ion binding & Down & 800 & 200 & Down \\
\hline AT5G60970 & TCP5; transcription factor & Down & 600 & 200 & Down \\
\hline
\end{tabular}

${ }^{\mathrm{a}} \mathrm{P} / \mathrm{C}$ : Direction of H3K27me3 change in primed versus non-primed plants.

${ }^{b} 24 \mathrm{~h}$ : Immediately after the priming treatment.

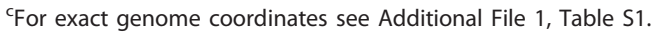

${ }^{\mathrm{d}} 10 \mathrm{~d}$ : After 10 days of growth in control conditions. 


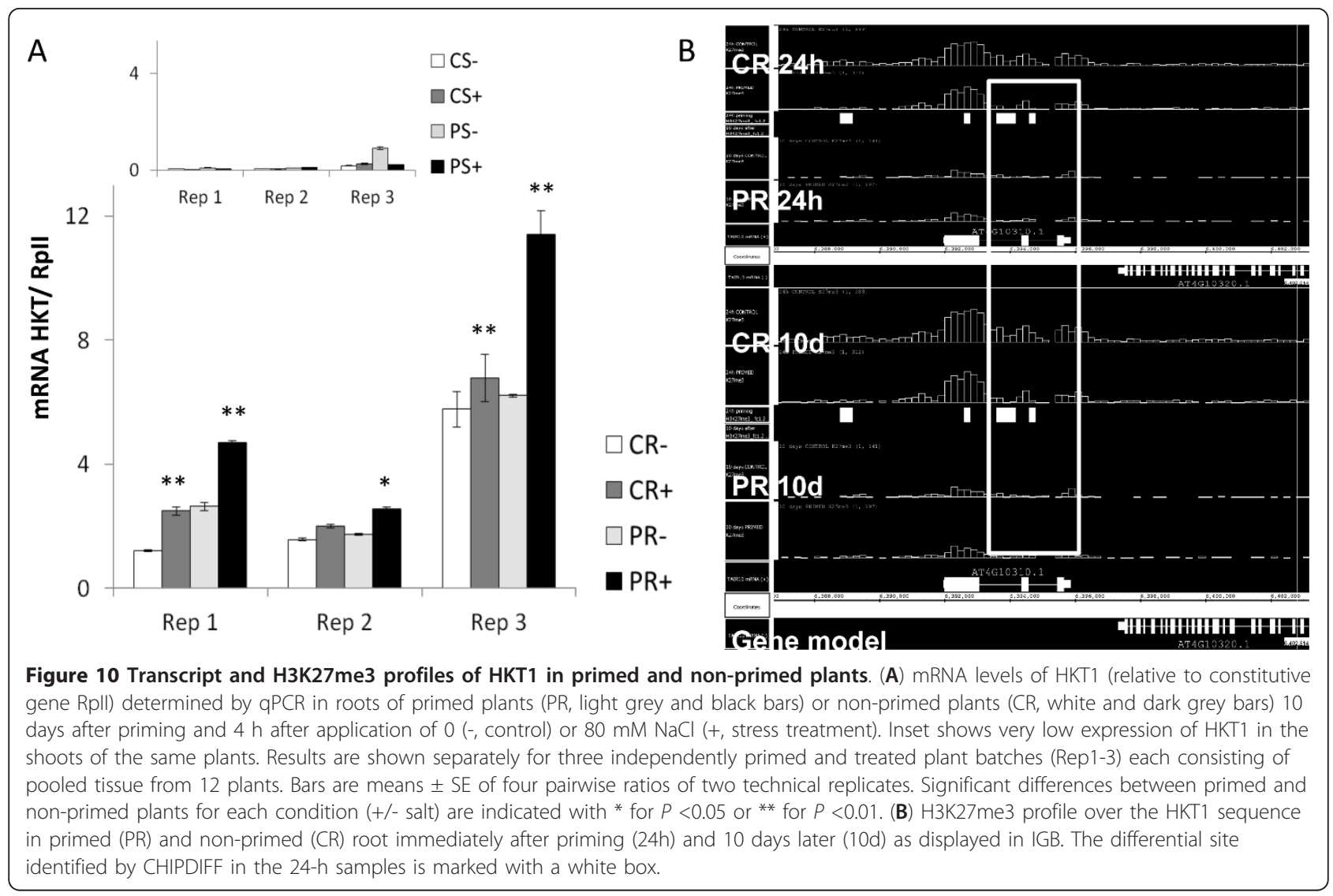

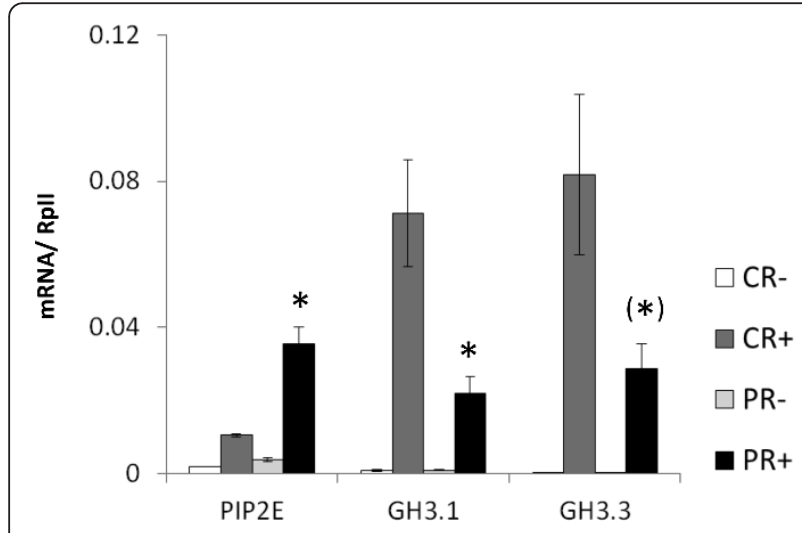

Figure 11 Transcript profiles of PIP2E, GH3.1, and GH3.3 in primed and non-primed plants. mRNA levels of PIP2E, GH3.1, and GH3.3 (relative to constitutive gene Rpll) determined by qPCR in roots of primed plants (PR, light grey and black bars) or non-primed plants (CR, white and dark grey bars) 10 days after priming and $4 \mathrm{~h}$ after application of $0(-)$ or $80 \mathrm{mM} \mathrm{NaCl}(+)$. Bars are means \pm SE of three independently treated replicate plant batches each consisting of 12 plants. Significant differences between primed and nonprimed plants for each condition (+/- salt) are indicated with * for $P$ $<0.05$ and $(*)$ for $P=0.06$. produces clean root material to enable identification of those genes that underlie the main priming effect of enhanced drought tolerance (Figure 2C, D).

\section{Conclusions}

A short treatment of $A$. thaliana seedlings with a moderate concentration of $\mathrm{NaCl}$ alters physiological responses of the adult plants to salt and drought stress, and causes small but significant changes in genomewide profiles of four histone modifications. Primingindued 'etching' of H3K27me3 islands is still apparent after a growth period of 10 days. The number of location-specific changes of H3K27me3 decreases during this period suggesting that the memory fades over time. Nevertheless persistent differences of H3K27me3 occur in $>100$ genes. One of these genes, encoding the $\mathrm{Na}-$ transporter HKT1, is more strongly induced in the primed plants than in the non-primed plants during a second salt treatment, which could explain the observed reduction of $\mathrm{Na}$ accumulation in salt-primed plants. The high-resolution genome-wide datasets generated in this study provide an essential resource for further exploration of the novel molecular features of somatic longterm memory in plants unveiled by our experiments. 


\section{Materials and methods \\ Plant growth and treatments}

Batches of approximately 300 Arabidopsis thaliana (Col0 ) seeds were germinated on vertical agar plates containing $70 \mathrm{~mL}$ of a minimally sufficient nutrient solution [55] supplemented with $3 \%$ sucrose (10 h light/14 h dark photoperiod, light intensity $120 \mu \mathrm{mol} \mathrm{m}^{-2} \mathrm{~s}^{-1}, 22^{\circ} \mathrm{C}$ ). When seedlings had reached the four-leaf stage $5 \mathrm{~mL}$ of growth medium supplemented with $50 \mathrm{mM} \mathrm{NaCl}$ (or other concentrations as stated in the Results section) was applied directly to the roots (priming treatment). Control plants were treated in the same way using nutrient medium without $\mathrm{NaCl}$ added. Twenty-four hours later seedlings were transferred either to soil or to hydroponics. After 10 days of growth in control conditions, watering of soil-grown plants was stopped to induce drought stress. At the same time $80 \mathrm{mM} \mathrm{NaCl}$ was added to the hydroponics to induce salt stress. Plant tissues were harvested at several times during the protocol as indicated in the Results section.

\section{Measurement of tissue ion content}

Ions were acid extracted from dried plant material by 24-h incubation with $2 \mathrm{M} \mathrm{HCl}(1: 100 \mathrm{w}: \mathrm{v})$. The Na concentration in the extraction buffer was measured by spectrophotometry (410 flame photometer, SherwoodScientific Ltd., Cambridge, UK) and related to the dry weight of the plant material used.

\section{Extraction and immunoprecipitation of chromatin}

Chromatin extraction and immunoprecipitation (ChIP) were carried out following published protocols [56]. In brief, tissue samples were incubated in $1 \%(\mathrm{w} / \mathrm{v})$ formaldehyde for 15 min under vacuum. Cross-linking was stopped by adding $125 \mathrm{mM}$ glycine, and tissues were rinsed, blotted dry, and frozen. Diluted chromatin extracts were incubated with antibodies against H3K4me2 (Diagenode pAb-035-050), H3K4me3 (Diagenode pAb-003-050), H3K9me2 (17-681, Millipore), or H3K27me3 (Diagenode, pAb-069-050). Immunoprecipitated chromatin-DNA (IPDNA) or input chromatin-DNA was reverse cross-linked and residual protein was removed by proteinase $\mathrm{K}$ treatment. DNA was recovered by phenol/chloroform extraction and ethanol precipitation. As a quality control for successful ChIP, existence or absence of sequences previously found to be associated (positive control) or not (negative control) with certain histone modifications $[32,34,57]$ in the ChIP samples was confirmed by semiquantitative PCR. The primer pairs listed in Additional file 1 , Table S3, recognized specific regions in the following genes: AT5G56920 (positive control) and AT5G56900 (negative control) for H3K27me3, AT1G24560 (positive and negative control) for H3K4me2/3, AT1G37110 (positive control) and AT2G05920 (negative control) for H3K9me2. Samples that had passed the quality control were used for further analyses.

\section{ChIP-qPCR and ChIP-Sequencing}

Primers were designed to amplify specific regions of interest as stated in the Results section. Primer pairs are listed in Additional file 1, Table S3. ChIP DNA and input DNA samples were linearly amplified using GenomePlex Complete Whole Genome Amplification (WGA2, Sigma-Aldrich) following the manufacture instructions. Amplified samples were used as template for qPCR analysis carried out with Brilliant III SYBR Green qPCR kit (Stratagene) on a Mx3000 system (Stratgene). Ct values obtained were dually normalized to input and to reference region (same as 'positive controls' in quality control, see above). Sequencing of the ChIP DNA was carried out in the Glasgow Polyomics Facility (University of Glasgow). A DNA library was prepared using the ChIP-SEQ Sample Prep Kit (Illumina), according to the manufacturer's protocol, size selected on an agarose gel, amplified by PCR, and loaded onto separate lanes of GAIIx flow cells at a concentration of $12 \mathrm{pM}$. After cluster formation (Illumina Cluster station) the samples were sequenced (Illumina Genome Analyzer IIx) producing single $76 \mathrm{bp}$ reads.

\section{mRNA extraction, RT-qPCR, and RNA-sequencing}

RNA was extracted with RNeasy mini kit (Qiagen) according to the manufacture instructions and reverse transcribed to cDNA using QuantiTect ${ }^{\mathbb{B}}$ Reverse Transcription kit (Qiagen). qPCR analysis were carried out as described above. Several conventionally used reference genes were evaluated for stable expression across our experimental conditions using geNorm [58] and on the basis of this analysis RpII (At4g35800) was chosen as reference gene. Sequencing of the RNA was carried out in the Glasgow Polyomics Facility. RNA-seq libraries were prepared using mRNA 8-Sample Prep Kit (Illumina). Briefly, polyA RNA was isolated from $1 \mu \mathrm{g}$ of total RNA, fragmented, and subjected to first- and second-strand cDNA synthesis with random primers. Subsequent sequencing procedures were the same as for DNA (see above).

\section{Analysis of ChIP-seq and RNA-seq data}

Read alignment: For each sample, unaligned 76 bp reads in fastq format were generated with Casava version 1.7 (Illumina Inc.). Reads were aligned to the A.thaliana genome (TAIR9) using Bowtie version 0.12.5 [59] allowing for either unique or multiple read alignments with up to two mismatches in the first 54 bases. The alignment files in SAM/BAM format were then sorted, and 
duplicated reads with the same orientation removed using Samtools [59]. Alignment of sequence reads to unique targets in the Arabidopsis thaliana genome accommodated $76 \%$ to $94 \%$ of sequences for H3K4me2, $\mathrm{H} 3 \mathrm{~K} 4 \mathrm{me} 3$, and H3K27me3, increasing to $79 \%$ to $97 \%$ when alignment to multiple targets was permitted. Only $42 \%$ of H3K9me 2 reads could be aligned to unique targets, but due to preferential association of H3K9me2 with repeat sequences this number increased to over $74 \%$ when multiple alignments were included. Subsequent analyses were based on unique alignments for H3K4me2, H3K4me3, and H3K27me3, and on multiple alignments for $\mathrm{H} 3 \mathrm{~K} 9 \mathrm{me} 2$. The final alignment positions of non-redundant reads were stored in BED files.

Identification of histone modification domains: Enriched histone modification domains ('islands') were calculated for each sample using SICER [35] version 1.03 with random background option (SICER-rb). A window size of $200 \mathrm{bp}$, an e-value of 0.1, and samplespecific DNA fragment sizes varying from 216 to $268 \mathrm{bp}$ were used throughout the calculations. Based on a simulated alignment by Bowtie of 76-bp reads, synthetically generated by exhaustive partition of the entire genome, an effective genome fraction of 0.94 was used in all calculations. To determine the optimal internal gap length, SICER-rb was run with gaps of 0-5 window sizes (0$1,000 \mathrm{bp}$ ). For each histone modification the gap length producing the maximal aggregate score of all significant islands was then selected for further calculations. The optimal gap lengths were 0 for $\mathrm{H} 3 \mathrm{~K} 4 \mathrm{me} 2$ and H3K4me3, 600 bp for H3K9me2, and $400 \mathrm{bp}$ for H3K27me3. SICER scores for each 200-bp window were stored in WIG files (Additional files 3 to 16). The genomic coordinates of the obtained islands were stored in BED files (Additional files 17 to 30). Both file formats can be displayed in modern genome browsers.

Identification of sites showing difference in histone modification levels: To identify histone modification sites that differed between primed and non-primed samples we used CHIPDIFF software based on a hidden Markov model approach [36]. Throughout the calculations we used the same values for effective genome fraction, sample-specific fragment sizes and optimized window sizes as in the SICER-rb calculations (see above). Applying these parameters required a small change in the software code. For other parameters default values were used [36]. Separate lists of differences were generated using thresholds of 1.2, 1.5, and 2fold changes. The genomic coordinates of the identified differences were stored in BED files. Files containing coordinates of $>1.2$-fold differences are provided as additional files for upload into genome browsers
(Additional files 31 to 37). A BED file containing coordinates of $A$. thaliana genes (TAIR9 version) is also provided for upload into genome browser (Additional File 38).

Mapping: The identified islands and differences were mapped onto TAIR9 gene annotations using an inhouse built script. A region was associated with a gene if its genomic coordinates overlapped with those of a gene including up- and downstream sequences of either $100 \mathrm{bp}$ or $1,000 \mathrm{bp}$ (separate lists). If two genes were assigned to an island/ the annotation of the closer one was selected. Lists of all mapped islands and differences were saved in Excel format for further analysis.

Analysis of RNA-seq data: From each sample unaligned single-end $76 \mathrm{bp}$ reads in fastq format were generated with Casava version 1.7 (Illumina Inc.). Reads were aligned to the A.thaliana genome (TAIR9) using Tophat version 1.4.0 [60]. Differences in mRNA-levels between primed and non-primed samples were explored with Cuffdiff version 1.3.0 [61] using upper-quartile normalization, which resulted in a list of genes with mRNA levels (in FPKM), fold changes (log2 of FPKM-ratio), and statistical significance in form of false discovery rate. For subsequent analyses the list was filtered for FDR $>0.05$ and $\log 2$ (FPKM ratio) $>1$.

\section{Primary accession}

ChIP-Seq and RNA-Seq raw data obtained in this study are available at ArrayExpress under series accession number E-MTAB-1663 and E-MTAB-1668, respectively.

\section{Description of additional files}

The following additional data are available with the online version of this paper: Additional file 1 is a pdf file containing Figures S1-4 and Tables S1-3. Figure S1 shows plants after long-term salt stress. Figure S2 presents the results of successful ChIP quality control. Figure S3 displays genome-wide histone modification landscapes. Figure S4 shows the kinetics of RNA and H3K27me3 levels for all genes tested. Table S1 details chromosome coordinates of priming-induced H3K27me3 differences recorded immediately after the $24-\mathrm{h}$ priming treatment and 10 days later. Table S2 lists the genes that were tested for transcriptional changes after the second salt treatment. Table S3 provides sequences of all primers used in this study. Additional file 2 is a .xls file containing all results of functional enrichments as obtained by DAVID. Additional files 3, 4, 5, 6, 7, 8, 9, 10, 11, 12, 13, $14,15,16,17,18,19,20,21,22,23,24,25,26,27,28$, $29,30,31,32,33,34,35,36,37$ provide genome-wide histone modification profiles that can be uploaded into any modern genome browser (for example, IGB). 
Additional files 3, 4, 5, 6, 7, 8, 9, 10, 11, 12, 13, 14, 15, 16 are .wig files containing read counts over 200-bp windows for all histone modifications in all samples. Additional files 17, 18, 19, 20, 21, 22, 23, 24, 25, 26, 27, 28, 29, 30 are .bed files containing genome coordinates of identified islands for all histone modifications in all samples. Additional files 31, 32, 33, 34, 35, 36, 37 are .bed files containing genome co-ordinates of identified differences (primed/non-primed) for all histone modifications in all samples. The labeling of these files is self-explanatory (C: Non-primed; P: Primed; R: Roots; S: Shoots). All files are for 24-h samples, except those labeled 'Tendays'. Additional file 38 contains coordinates of $A$. thaliana Col-0 genes (TAIR9 version).

\section{Additional material}

Additional file 1: Additional Figures and Table. Figure S1. Appearance of primed and non-primed plants after long-term salt stress. Plants had been treated with either 0 (control, not primed, left), $50 \mathrm{mM}$ (primed, center), or $100 \mathrm{mM} \mathrm{NaCl}$ (primed, right) for $24 \mathrm{~h}$ at seedling stage and subsequently grown for 10 days in hydroponics without salt. The solution was then supplemented with $80 \mathrm{mM}$ or not (control, no salt) and plants photographed 10 days later. No difference in salt tolerance was apparent between primed and non-primed plants. Figure S2. Example of a successful ChIP quality control. Based on published histone methylation profiles primers were designed to amplify regions that are enriched (positive controls) or devoid (negative controls) of H3K4me2, H3K4me3, H3K9me2, or H3K4me3. (Note that no region was found that was exclusively associated with H3K4me2.) ChIP samples from roots (R) or shoots (S) of primed (50) or non-primed (C) plants obtained with antibody against H3K9me2 (A), H3K27me3 (A), H3K4me2 (M2), or H3K4me3 (M3) were used as template as well as ChIP input DNA (I) and ChIP without antibodies (NA). For primer pairs see Table S3. Figure S3. Genome-wide histone modification landscapes in primed and nonprimed plants. Genome-wide profiles of read counts for H3K4me2 (green), H3K4me3 (red), H3K9me2 (purple), and H3K27me3 (blue) in roots samples of primed (PR) and non-primed (CR) plants displayed in the Integrated Genome Browser (IGB). Figure S4. Kinetics of H3K27me3 and mRNA after salt application. Relative enrichment of H3K27me3 (black bars, left $y$-axis) and mRNA levels (open bars, right $y$-axis) of nine genes in roots of $A$. thaliana seedlings were determined by GPCR over a time course of $8 \mathrm{~h}$ (x-axis) after application of $50 \mathrm{mM} \mathrm{NaCl}$ (priming treatment). H3K27me3 levels (left y-axis) were normalized to ChIP input and to reference region in At5g56920. mRNA levels (right $y$-axis) were normalized to reference gene Rpll. For details see main text and Figure 6D. Table S1. Chromosome coordinates of priming-induced H3K27me3 differences. Table S2. Expression levels of selected genes in root RNA $4 \mathrm{~h}$ after second salt treatment. Table S3. Sequences of primers used in this study

Additional file 2: Enrichment of functional terms among genes with mapped differences in $\mathrm{H} 3 \mathrm{~K} 4 \mathrm{me} 3$ and $\mathrm{H} 3 \mathrm{~K} 27 \mathrm{me} 3$ as determined by DAVID. Enrichment calculated over genes with mapped islands in control (background C) or primed samples (background P). Upstream and downstreams sequences included were either $100 \mathrm{bp}$ or $100 \mathrm{pb}$. Additional file 3: Reads200_CRH3K4me2.wig. WIG file containing sequence read counts over $200 \mathrm{bp}$ windows in sample 24h CRH3K4me2. Additional file 4: Reads200_CRH3K4me3.wig. WIG file containing sequence read counts over $200 \mathrm{bp}$ windows in sample 24h CRH3K4me3 Additional file 5: Reads200_CRH3K9me2.wig. WIG file containing sequence read counts over 200 bp windows in sample 24h CRH3K9me2
Additional file 6: Reads200_CRH3K27me3.wig. WIG file containing sequence read counts over 200 bp windows in sample 24h CRH3K27me3 Additional file 7: Reads200_CSH3K4me2.wig. WIG file containing sequence read counts over $200 \mathrm{bp}$ windows in sample 24h CSH3K4me2 Additional file 8: Reads200_CSH3K4me3.wig. WIG file containing sequence read counts over 200 bp windows in sample 24h CSH3K4me3 Additional file 9: Reads200_PRH3K4me2.wig. WIG file containing sequence read counts over 200 bp windows in sample 24h PRH3K4me2 Additional file 10: Reads200_PRH3K4me3.wig. WIG file containing sequence read counts over 200 bp windows in sample 24h PRH3K4me3 Additional file 11: Reads200_PRH3K9me2.wig. WIG file containing sequence read counts over 200 bp windows in sample 24h PRH3K9me2 Additional file 12: Reads200_PRH3K27me3.wig. WIG file containing sequence read counts over $200 \mathrm{bp}$ windows in sample 24h PRH3K27me3 Additional file 13: Reads200_PSH3K4me2.wig. WIG file containing sequence read counts over 200 bp windows in sample 24h PSH3K4me2 Additional file 14: Reads200_PSH3K4me3.wig. WIG file containing sequence read counts over 200 bp windows in sample 24h PSH3K4me3 Additional file 15: Reads200_TendaysCRH3K27me3.wig. WIG file containing sequence read counts over $200 \mathrm{bp}$ windows in sample $24 \mathrm{~h}$ CRH3K27me3

Additional file 16: Reads200_TendaysPRH3K27me3.wig. WIG file containing sequence read counts over $200 \mathrm{bp}$ windows in sample 10d PRH3K27me3

Additional file 17: Islands_CRH3K4me2-G600.bed. BED file containing coordinates of islands as determined by SICER software in sample $24 \mathrm{~h}$ CRH3K4me2. (G: optimized gap size in bp.)

Additional file 18: Islands_CRH3K4me3-G600.bed. BED file containing coordinates of islands as determined by SICER software in sample $24 \mathrm{~h}$ CRH3K4me3. (G: optimized gap size in bp.)

Additional file 19: Islands_CRH3K9me2-G400.bed. BED file containing coordinates of islands as determined by SICER software in sample $24 \mathrm{~h}$ CRH3K9me2. (G: optimized gap size in bp.)

Additional file 20: Islands_CRH3K27me3-G600.bed. BED file containing coordinates of islands as determined by SICER software in sample 24h CRH3K27me3. (G: optimized gap size in bp.)

Additional file 21: Islands_CSH3K4me2-G600.bed. BED file containing coordinates of islands as determined by SICER software in sample $24 \mathrm{~h}$ CSH3K4me2. (G: optimized gap size in bp.)

Additional file 22: Islands_CSH3K4me3-G600.bed. BED file containing coordinates of islands as determined by SICER software in sample $24 \mathrm{~h}$ CSH3K4me2. (G: optimized gap size in bp.)

Additional file 23: Islands_PRH3K4me2-G600.bed. BED file containing coordinates of islands as determined by SICER software in sample $24 \mathrm{~h}$ PRH3K4me2. (G: optimized gap size in bp.)

Additional file 24: Islands_PRH3K4me3-G600.bed. BED file containing coordinates of islands as determined by SICER software in sample $24 \mathrm{~h}$ PRH3K4me3. (G: optimized gap size in bp.)

Additional file 25: Islands PRH3K9me2-G400.bed. BED file containing coordinates of islands as determined by SICER software in sample $24 \mathrm{~h}$ PRH3K9me2. (G: optimized gap size in bp.)

Additional file 26: Islands_PRH3K27me3-G600.bed. BED file containing coordinates of islands as determined by SICER software in sample 24h PRH3K27me3. (G: optimized gap size in bp.)

Additional file 27: Islands_PSH3K4me2-G600.bed. BED file containing coordinates of islands as determined by SICER software in sample $24 \mathrm{~h}$ PSH3K4me2. (G: optimized gap size in bp.)

Additional file 28: Islands_PSH3K4me3-G600.bed. BED file containing coordinates of islands as determined by SICER software in sample $24 \mathrm{~h}$ PSH3K4me3. (G: optimized gap size in bp.) 
Additional file 29: slands_TendaysCRH3K27me3-G400.bed. BED file containing coordinates of islands as determined by SICER software in sample 10d CRH3K27me3. (G: optimized gap size in bp.)

Additional file 30: Islands_TendaysPRH3K27me3-G400.bed. BED file containing coordinates of islands as determined by SICER software in sample 10d PRH3K27me3. (G: optimized gap size in bp.)

Additional file 31: Differences_PvsC_RH3K4me2.bed. BED file containing coordinates for differences determined by CHIPDIFF software between primed (P) and non-primed (C) 24h RH3K4me2 samples.

Additional file 32: Differences PvsC RH3K4me3.bed. BED file containing coordinates for differences determined by CHIPDIFF software between primed (P) and non-primed (C) 24h RH3K4me3 samples.

Additional file 33: Differences_PvsC_RH3K9me2.bed. BED file containing coordinates for differences determined by CHIPDIFF software between primed (P) and non-primed (C) 24h RH3K9me2 samples.

Additional file 34: Differences_PvsC_RH3K27me3.bed. BED file containing coordinates for differences determined by CHIPDIFF software between primed (P) and non-primed (C) 24h RH3K27me3 samples.

Additional file 35: Differences_PvsC_SH3K4me2.bed. BED file containing coordinates for differences determined by CHIPDIFF software between primed (P) and non-primed (C) 24h SH3K4me2 samples.

Additional file 36: Differences_PvsC_SH3K4me3.bed. BED file containing coordinates for differences determined by CHIPDIFF software between primed (P) and non-primed (C) 24h SH3K4me3 samples.

Additional file 37: Differences_PvsC_TendaysRH3K27me3.bed. BED file containing coordinates for differences determined by CHIPDIFF software between primed (P) and non-primed (C) 10d RH3K27me3 samples.

Additional file 38: TAIR9genes.bed. BED file containing coordinates of A. thaliana genes.

\section{Abbreviations}

AG8: AGAMOUS 8; AGI: Arabidopsis Genome Initiative; ATGOLS3: Arabidopsis Thaliana Galactinol Synthase 3; ATX1: Arabidopsis homolog of Trithorax 1; BAM: Binary Alignment/Map; BED: Browser Extensible Data; bp: Base pair; ChIP: Chromatin Immuno-precipitation; ChIP-qPCR: ChIP followed by QPCR; ChIP-Seq: ChIP Chromatin followed by Sequencing; Col-0: Columbia-0; COR15A: Cold-regulated 15A; Ct: Cycle threshold; CR: Control roots; CYP71B4: Cytochrome P450 family71B4; DAVID: Database for Annotation Visualization and Integrated Discovery; DNA: Deoxyribonucleic acid; DW: Dry weight; FDR: False detection rate; FPKM: Number of RNA fragments per kilobase of gene model per million of aligned reads; FW: Fresh weight; GH3: Gretchen Hagen 3; GGPS4: Geranylgeranyl pyrophosphate synthase 4; H3K4: Lysine 4 in histone 3; H3K27: Lysine 27 in histone 3; H3K4me2: Histone H3 dimethylated at lysine 4; H3K4me3: Histone H3 tri-methylated at lysine 4; H3K27me3: Histone H3 tri-methylated at lysine 27; H3K9me2: Histone H3 dimethylated at lysine 9; HKT1: High-affinity K transporter 1; HMT: Histone methyl-transferase; IGB: Integrated genome browser; LRP1: Low response to phosphate 1; mRNA: messenger Ribo-Nucleic Acid; MYB: Myeloblastosis family; NaCl: Sodium chloride; nt: Nucleotide; GPCR: quantitative polymerase chain reaction; $P$ value: Probability value; PIP2E: Plasma membrane intrinsic protein 2E; pM: pico-Molar; RNA-Seq; RNA isolation followed by sequencing; polyA: Polyadenylated; PR: Primed roots; PRC1: Polycomb repressive complex 1; REF6: Relative of early flowering 6; Rpll: RNA polymerase II; RLP43: Receptor like protein 43; RT-PCR: Reverse-transcribed polymerase chain reaction; siRNA: Small interfering RNA; SAM: Sequence alignment/map; SE: Standard error; SHP1: Shatterproof 1; SOS5: Salt overly-sensitive 5; SP-PIR KEYWORDS: Swiss-Prot-Protein Information Resource Keywords; SUVH: Suppressor of variegation H; TAIR: The Arabidopsis Information Resources; TEL1: Terminal Ear1-Like 1; WGA: Whole genome amplification; WIG: Wiggle track format; $w / v$ : Weight per volume.

\section{Authors' contributions}

ES carried out all experimental work apart from RT-qPCR analysis of transcriptional responses to salt stress, which was carried out by GP. PH generated and analyzed the ChIP-Seq and RNA-Seq data. VC participated in project design, co-supervised the experimental work, and helped to draft the manuscript. AA designed and managed the project, and wrote the manuscript. All authors have read and approved the final manuscript.

\section{Competing interests}

The authors declare that they have no competing interests.

\section{Acknowledgements}

We would like to thank George Boswell, Liz O'Donnell, Julie Galbraith, and Jing Wang for technical assistance. We are grateful to the Leverhulme Trust for funding this project (F/00179/AS)

\section{Author details}

${ }^{1}$ Institute of Molecular, Cell and Systems Biology (MCSB), College of Medical, Veterinary \& Life Sciences (MVLS), University of Glasgow, Glasgow G128QQ, UK. ${ }^{2}$ Ecole Normale Supérieure, Institut de Biologie de I'ENS (IBENS), Paris, F75005, France. ${ }^{3}$ Institute of Cell Biology, University of Edinburgh, Edinburgh EH9 3JR, UK.

Received: 19 March 2013 Revised: 21 May 2013

Accepted: 14 June 2013 Published: 14 June 2013

\section{References}

1. Conrath U: Molecular aspects of defence priming. Trends Plant Sci 2011, 16:524-531.

2. Ventura L, Dona M, Macovei A, Carbonera D, Buttafava A, Mondoni A, Rossi G, Balestrazzi A: Understanding the molecular pathways associated with seed vigor. Plant Physiol Biochem 2012, 60:196-206.

3. Sung DY, Kaplan F, Lee KJ, Guy CL: Acquired tolerance to temperature extremes. Trends Plant Sci 2003, 8:179-187.

4. Roudier F, Teixeira FK, Colot V: Chromatin indexing in Arabidopsis: an epigenomic tale of tails and more. Trends Genet 2009, 25:511-517.

5. Vaillant I, Paszkowski J: Role of histone and DNA methylation in gene regulation. Curr Opin Plant Biol 2007, 10:528-533.

6. Johannes F, Porcher E, Teixeira FK, Saliba-Colombani V, Simon M, Agier N, Bulski A, Albuisson J, Heredia F, Audigier P, Bouchez D, Dillmann C, Guerche P, Hospital F, Colot V: Assessing the impact of transgenerational epigenetic variation on complex traits. PLOS Genet 2009, 5:e1000530.

7. Hauben M, Haesendonckx B, Standaert E, Van Der Kelen K, Azmi A, Akpo H, Van Breusegem F, Guisez Y, Bots M, Lambert B, Laga B, De Block M: Energy use efficiency is characterized by an epigenetic component that can be directed through artificial selection to increase yield. Proc Natl Acad Sci USA 2009, 106:20109-20114.

8. Bilichak A, Inystkyy Y, Hollunder J, Kovalchuk I: The progeny of Arabidopsis thaliana plants exposed to salt exhibit changes in DNA methylation, histone modifications and gene expression. PLoS One 2012, 7:e30515.

9. Luna E, Bruce TJA, Roberts MR, Flors V, Ton J: Next-generation systemic acquired resistance. Plant Physiol 2012, 158:844-853.

10. Lang-Mladek C, Popova O, Kiok K, Berlinger M, Rakic B, Aufsatz W, Jonak C, Hauser MT, Luschnig C: Transgenerational inheritance and resetting of stress-induced loss of epigenetic gene silencing in Arabidopsis. Mol Plant 2010, 3:594-602.

11. Verhoeven KJF, Jansen JJ, van Dijk PJ, Biere A: Stress-induced DNA methylation changes and their heritability in asexual dandelions. New Phytol 2010, 185:1108-1118.

12. Ou XF, Zhang $Y H, X u C M$, Lin $X Y$, Zang $Q$, Zhuang $T$, Jiang $L L$, von Wettstein D, Liu B: Transgenerational inheritance of modified DNA methylation patterns and enhanced tolerance induced by heavy metal stress in rice (Oryza sativa L.). PLoS One 2012, 7:e41143.

13. Ito H, Gaubert H, Bucher E, Mirouze M, Vaillant I, Paszkowski J: An siRNA pathway prevents transgenerational retrotransposition in plants subjected to stress. Nature 2011, 472:115-119.

14. Pecinka A, Scheid OM: Stress-induced chromatin changes: a critical view on their heritability. Plant Cell Physiol 2012, 53:801-808.

15. Becker $C$, Weigel $D$ : Epigenetic variation: origin and transgenerational inheritance. Curr Opin Plant Biol 2012, 15:562-567.

16. Schmitz RJ, Schultz MD, Lewsey MG, O'Malley RC, Urich MA, Libiger O, Schork NJ, Ecker JR: Transgenerational epigenetic instability is a source of novel methylation variants. Science 2011, 334:369-373. 
17. Zhang YY, Fischer $M$, Colot V, Bossdorf O: Epigenetic variation creates potential for evolution of plant phenotypic plasticity. New Phytol 2013, 197:314-322.

18. Weigel D, Colot V: Epialleles in plant evolution. Genome Biol 2012, 13:249.

19. Angel A, Song J, Dean C, Howard M: A Polycomb-based switch underlying quantitative epigenetic memory. Nature 2011, 476:105-108.

20. Jaskiewicz $\mathrm{M}$, Conrath U, Peterhansel C: Chromatin modification acts as a memory for systemic acquired resistance in the plant stress response. Embo Reports 2011, 12:50-55.

21. Dowen RH, Pelizzola M, Schmitz RJ, Lister R, Dowen JM, Nery JR, Dixon JE, Ecker JR: Widespread dynamic DNA methylation in response to biotic stress. Proc Natl Acad Sci USA 2012, 109:E2183-E2191.

22. Yu A, Lepère G, Jay F, Wang J, Bapaume L, Wang Y, Abraham A-L, Penterman J, Fischer RL, Voinnet O, Navarro L: Dynamics and biological relevance of DNA demethylation in Arabidopsis antibacterial defense. Proc Natl Acad Sci USA 2013, 110:2389-2394.

23. Zong W, Zhong XC, You J, Xiong LZ: Genome-wide profiling of histone H3K4-tri-methylation and gene expression in rice under drought stress. Plant Mol Biol 2013, 81:175-188.

24. Kim JM, To TK, Ishida J, Morosawa T, Kawashima M, Matsui A, Toyoda T, Kimura $\mathrm{H}$, Shinozaki K, Seki M: Alterations of lysine modifications on the histone $\mathrm{H} 3 \mathrm{~N}$-tail under drought stress conditions in Arabidopsis thaliana. Plant Cell Physiol 2008, 49:1580-1588.

25. Dijk Kv, Ding Y, Malkaram S, Riethoven JJM, Liu R, Yang JY, Laczko P, Chen $H_{\text {, }}$ Xia YN, Ladunga I, Avramova Z, Fromm M: Dynamic changes in genomewide histone $\mathrm{H} 3$ lysine 4 methylation patterns in response to dehydration stress in Arabidopsis thaliana. BMC Plant Biology 2010, 10:238.

26. Kwon CS, Lee D, Choi G, Chung Wl: Histone occupancy-dependent and -independent removal of H3K27 trimethylation at cold-responsive genes in Arabidopsis. Plant J 2009, 60:112-121.

27. Brusslan JA, Alvarez-Canterbury AMR, Nair NU, Rice JC, Hitchler MJ, Pellegrini M: Genome-wide evaluation of histone methylation changes associated with leaf senescence in Arabidopsis. PLoS One 2012, 7:e33151

28. Ding Y, Avramova Z, Fromm M: Two distinct roles of ARABIDOPSIS HOMOLOG OF TRITHORAX1 (ATX1) at promoters and within transcribed regions of ATX1-regulated genes. Plant Cell 2011, 23:350-363.

29. Roudier F, Ahmed I, Berard C, Sarazin A, Mary-Huard T, Cortijo S, Bouyer D, Caillieux E, Duvernois-Berthet E, Al-Shikhley L, Giraut L, Despres B, Drevensek S, Barneche F, Derozier S, Brunaud V, Aubourg S, Schnittger A, Bowler C, Martin-Magniette ML, Robin S, Caboche M, Colot V: Integrative epigenomic mapping defines four main chromatin states in Arabidopsis. EMBO J 2011, 30:1928-1938

30. Liu CY, Lu FL, Cui X, Cao XF: Histone Methylation in Higher Plants. In Annual Review of Plant Biology. Volume 61. Edited by: Merchant S, Briggs WR, Ort D. Palo Alto, CA: Annual Reviews; 2010:395-420.

31. Nicol JW, Helt GA, Blanchard SG, Raja A, Loraine AE: The Integrated Genome Browser: free software for distribution and exploration of genome-scale datasets. Bioinformatics 2009, 25:2730-2731.

32. Zhang XY, Clarenz O, Cokus S, Bernatavichute $\mathrm{YV}$, Pellegrini M, Goodrich J, Jacobsen SE: Whole-genome analysis of histone H3 lysine 27 trimethylation in Arabidopsis. PLoS Biol 2007, 5:1026-1035.

33. Bernatavichute $Y$, Zhang XY, Cokus S, Pellegrini M, Jacobsen SE: Genomewide association of histone $\mathrm{H} 3$ lysine nine methylation with CHG DNA methylation in Arabidopsis thaliana. PLoS One 2008, 8:e3156.

34. Zhang XY, Bernatavichute $\mathrm{V}$, Cokus S, Pellegrini M, Jacobsen SE: Genomewide analysis of mono-, di- and trimethylation of histone $\mathrm{H} 3$ lysine 4 in Arabidopsis thaliana. Genome Biol 2009, 10:R62.

35. Zang CZ, Schones DE, Zeng C, Cui KR, Zhao KJ, Peng WQ: A clustering approach for identification of enriched domains from histone modification ChIP-Seq data. Bioinformatics 2009, 25:1952-1958.

36. $\mathrm{Xu} \mathrm{H}$, Wei $\mathrm{CL}$, Lin F, Sung WK: An HMM approach to genome-wide identification of differential histone modification sites from ChIP-seq data. Bioinformatics 2008, 24:2344-2349.

37. Song J, Angel A, Howard M, Dean C: Vernalization - a cold-induced epigenetic switch. J Cell Sci 2012, 125:3723-3731.

38. Margueron R, Justin N, Ohno K, Sharpe ML, Son J, Drury WJ, Voigt P, Martin SR, Taylor WR, De Marco V, Pirrotta V, Reinberg D, Gamblin SJ: Role of the polycomb protein EED in the propagation of repressive histone marks. Nature 2009, 461:762-767.
39. Lu FL, Cui X, Zhang SB, Jenuwein T, Cao XF: Arabidopsis REF6 is a histone H3 lysine 27 demethylase. Nat Genet 2011, 43:715-719.

40. Huang DW, Sherman BT, Lempicki RA: Systematic and integrative analysis of large gene lists using DAVID bioinformatics resources. Nat Prot 2009, 4:44-57.

41. Maathuis FJM, Filatov V, Herzyk P, Krijger GC, Axelsen KB, Chen SX, Green BJ, Li Y, Madagan KL, Sanchez-Fernandez R, Forde BG, Palmgren MG, Rea PA, Williams LE, Sanders D, Amtmann A: Transcriptome analysis of root transporters reveals participation of multiple gene families in the response to cation stress. Plant J 2003, 35:675-692.

42. Scharf AND, Barth TK, Imhof A: Establishment of histone modifications after chromatin assembly. Nucleic Acids Res 2009, 37:5032-5040.

43. Henikoff S, Shilatifard A: Histone modification: cause or cog? Trends Genet 2011, 27:389-396.

44. Han SK, Sang Y, Rodrigues A, Wu MF, Rodriguez PL, Wagner D, Biol F: The SWI2/SNF2 chromatin remodeling ATPase BRAHMA represses abscisic acid responses in the absence of the stress stimulus in Arabidopsis. Plant Cell 2012, 24:4892-4906.

45. Luo M, Liu XC, Singh P, Cui YH, Zimmerli L, Wu KQ: Chromatin modifications and remodeling in plant abiotic stress responses. Biochim Biophys Acta 2012, 1819:129-136.

46. Davenport RJ, Munoz-Mayor A, Jha D, Essah PA, Rus A, Tester M: The Na+ transporter AtHKT1;1 controls retrieval of $\mathrm{Na}+$ from the xylem in Arabidopsis. Plant Cell Environ 2007, 30:497-507.

47. Moller IS, Gilliham M, Jha D, Mayo GM, Roy SJ, Coates JC, Haseloff J, Tester M: Shoot $\mathrm{Na}+$ exclusion and increased salinity tolerance engineered by cell type-specific alteration of $\mathrm{Na}+$ transport in Arabidopsis. Plant Cell 2009, 21:2163-2178.

48. Berthomieu P, Conejero G, Nublat A, Brackenbury WJ, Lambert C, Savio C, Uozumi N, Oiki S, Yamada K, Cellier F, Gosti F, Simonneau T, Essah PA Tester M, Very AA, Sentenac H, Casse F: Functional analysis of AtHKT1 in Arabidopsis shows that $\mathrm{Na}^{+}$recirculation by the phloem is crucial for salt tolerance. EMBO J 2003, 22:2004-2014

49. Rus A, Lee BH, Munoz-Mayor A, Sharkhuu A, Miura K, Zhu JK, Bressan RA, Hasegawa PM: AtHKT1 facilitates Na+ homeostasis and $\mathrm{K}+$ nutrition in planta. Plant Physiol 2004, 136:2500-2511.

50. Baek D, Jiang JF, Chung JS, Wang BS, Chen JP, Xin ZG, Shi HZ: Regulated AtHKT1 gene expression by a distal enhancer element and DNA methylation in the promoter plays an important role in salt tolerance. Plant Cell Physiol 2011, 52:149-161.

51. Rus A, Baxter I, Muthukumar B, Gustin J, Lahner B, Yakubova E, Salt DE: Natural variants of AtHKT1 enhance $\mathrm{Na}(+)$ accumulation in two wild populations of Arabidopsis. PLoS Genet 2006, 2:e210.

52. Pradoa K, Boursiaca Y, Tournaire-Rouxa C, Monneuseb J-M, Postairea O, Da Inesc O, Schäffner AR, Hemb S, Santonia V, Maurel C: Regulation of Arabidopsis leaf hydraulics involves light-dependent phosphorylation of aquaporins in veins. Plant Cell 2013, 25:1029-1039.

53. Gutierrez L, Mongelard G, Flokova K, Pacurar DI, Novak O, Staswick P, Kowalczyk M, Pacurar M, Demailly H, Geiss G, Bellini C: Auxin controls Arabidopsis adventitious root initiation by regulating jasmonic acid homeostasis. Plant Cell 2012, 24:2515-2527.

54. Birnbaum K, Shasha DE, Wang JY, Jung JW, Lambert GM, Galbraith DW, Benfey PN: A gene expression map of the Arabidopsis root. Science 2003, 302:1956-1960.

55. Kellermeier F, Chardon F, Amtmann A: Natural variation of Arabidopsis root architecture reveals complementing adaptive strategies to potassium starvation. Plant Physiol 2013, 161:1421-1432.

56. Saleh A, Alvarez-Venegas R, Yilmaz M, Le O, Hou GC, Sadder M, AlAbdallat A, Xia YN, Lu GQ, Ladunga I, Avramova Z: The highly similar Arabidopsis homologs of trithorax ATX1 and ATX2 encode proteins with divergent biochemical functions. Plant Cell 2008, 20:568-579.

57. Zhou JL, Wang XF, He K, Charron JBF, Elling AA, Deng XW: Genome-wide profiling of histone $\mathrm{H} 3$ lysine 9 acetylation and dimethylation in Arabidopsis reveals correlation between multiple histone marks and gene expression. Plant Mol Biol 2010, 72:585-595.

58. Vandesompele J, De Preter K, Pattyn F, Poppe B, Van Roy N, De Paepe A, Speleman F: Accurate normalization of real-time quantitative RT-PCR data by geometric averaging of multiple internal control genes. Genome Biol 2002, 3:RESEARCH0034. 
59. Langmead B, Trapnell C, Pop M, Salzberg SL: Ultrafast and memoryefficient alignment of short DNA sequences to the human genome. Genome Biol 2009, 10:R25.

60. Trapnell C, Pachter L, Salzberg SL: TopHat: discovering splice junctions with RNA-Seq. Bioinformatics 2009, 25:1105-1111.

61. Trapnell C, Williams BA, Pertea G, Mortazavi A, Kwan G, van Baren MJ, Salzberg SL, Wold BJ, Pachter L: Transcript assembly and quantification by RNA-Seq reveals unannotated transcripts and isoform switching during cell differentiation. Nat Biotechnol 2010, 28:511-515.

doi:10.1186/gb-2013-14-6-r59

Cite this article as: Sani et al: Hyperosmotic priming of Arabidopsis seedlings establishes a long-term somatic memory accompanied by specific changes of the epigenome. Genome Biology 2013 14:R59.

Submit your next manuscript to BioMed Central and take full advantage of:

- Convenient online submission

- Thorough peer review

- No space constraints or color figure charges

- Immediate publication on acceptance

- Inclusion in PubMed, CAS, Scopus and Google Scholar

- Research which is freely available for redistribution

Submit your manuscript at www.biomedcentral.com/submit
(Ciomed Central 